The University of Southern Mississippi

The Aquila Digital Community

Honors Theses

Honors College

Spring 5-2015

\title{
Modeling the Diffusion of Heat Energy within Composites of Homogeneous Materials using the Uncertainty Principle
}

Elyse M. Garon

University of Southern Mississippi

Follow this and additional works at: https://aquila.usm.edu/honors_theses

Part of the Analysis Commons

\section{Recommended Citation}

Garon, Elyse M., "Modeling the Diffusion of Heat Energy within Composites of Homogeneous Materials using the Uncertainty Principle" (2015). Honors Theses. 311.

https://aquila.usm.edu/honors_theses/311

This Honors College Thesis is brought to you for free and open access by the Honors College at The Aquila Digital Community. It has been accepted for inclusion in Honors Theses by an authorized administrator of The Aquila Digital Community. For more information, please contact Joshua.Cromwell@usm.edu. 
The University of Southern Mississippi

Modeling the Diffusion of Heat Energy within Composites of Homogeneous Materials using the Uncertainty Principle

by

Elyse Marie Garon

\begin{abstract}
A Thesis
Submitted to the Honors College of The University of Southern Mississippi in Partial Fulfillment

of the Requirements for the Degree of Bachelor of Science

in the Department of Mathematics
\end{abstract}

May 2015 
Approved by

James V. Lambers, Ph.D., Thesis Adviser Associate Professor of Mathematics

Bernd Schroeder, Ph.D., Chair

Department of Mathematics

Ellen Weinauer, Ph.D., Dean

Honors College 


\begin{abstract}
The purpose of this project is to model the diffusion of heat energy in one space dimension, such as within a rod, in the case where the heat flow is through a medium consisting of two or more homogeneous materials. The challenge of creating such a mathematical model is that the diffusivity will be represented using a piecewise constant function, because the diffusivity changes based on the material. The resulting model cannot be solved using analytical methods, and is impractical to solve using existing numerical methods, thus necessitating a novel approach.

The approach presented in this thesis is to represent the solution as a linear combination of wave functions that change frequencies at the boundaries of different materials. It will be demonstrated that by using the Uncertainty Principle to construct a basis of such functions, in conjunction with a numerical method that is ideally suited to work with them, a mathematical model for heat diffusion through different materials can be solved much more efficiently than with other well-established methods from the literature.
\end{abstract}

Key Words: heat equation, Uncertainty Principle, spectral methods 


\section{Acknowledgments}

I would like to thank my advisor and mentor, Dr. James Lambers, for the guidance and support he has given me during my time at The University of Southern Mississippi. Furthermore, I would like to thank my supportive family and friends who were my constant cheerleaders and motivators. I am so grateful. 
Table of Contents

List of Tables . . . . . . . . . . . . . . . . . . . . . . . . viii

List of Illustrations . . . . . . . . . . . . . . . . . . . . . . ix

Chapter 1: Introduction . . . . . . . . . . . . . . . . . . . . . 1

Chapter 2: Literature Review . . . . . . . . . . . . . . . . . . 3

Chapter 3: Methodology . . . . . . . . . . . . . . . . . . . 6

Chapter 4: Finding the Eigenfunction . . . . . . . . . . . . . . . 11

Chapter 5: Solving the PDE . . . . . . . . . . . . . . . . . . . . . . . 19

Chapter6: Numerical Results . . . . . . . . . . . . . . . . . . 23

Finding Eigenfunctions . . . . . . . . . . . . . . . . . . . 23

Results from Solving the PDE . . . . . . . . . . . . . . . . 23

Chapter 7: Conclusion . . . . . . . . . . . . . . . . . 27

Appendix A: Code . . . . . . . . . . . . . . . . . . . . . . . 28

$G_{1}(\mathrm{G} 1 . \mathrm{m})$. . . . . . . . . . . . . . . . . 28

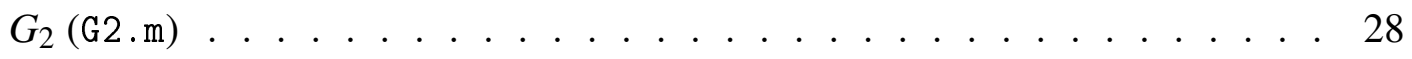

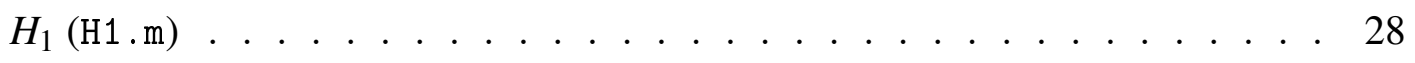

$H_{2}(\mathrm{H} 2 . \mathrm{m})$. . . . . . . . . . . . . . . . . . . . 29

Fourier Transform (myfft.m) _. . . . . . . . . . . . . . . . . . 29

Solving for $U_{j}$ (finalform.m)

Secant Method (secant.m) . . . . . . . . . . . . . . . . 31

Finding $\omega_{j 2}$ Frequencies (getw2 . m) . . . . . . . . . . . . . . . 32

Finding Eigenvalues (findeigs.m) . . . . . . . . . . . . . . . . 33 
Solve PDE (solvepde.m) . . . . . . . . . . . . . . . . 33

Bibliography .......................... 36 


\section{List of Tables}

6.1 Eigenvalues computed using eig, and using the method from Chapter 4, with $\alpha_{1}=1, \alpha_{2}=2$, and $\rho=3 / 8 \ldots \ldots \ldots \ldots \ldots$

6.2 Eigenvalues computed using eig, and using the method from Chapter 4, with $\alpha_{1}=1, \alpha_{2}=2$, and $\rho=\sqrt{2} /(2 \pi) \ldots \ldots \ldots \ldots 24$

6.3 Eigenvalues computed using eig, and using the method from Chapter 4 , with $\alpha_{1}=1, \alpha_{2}=4$, and $\rho=3 / 8 \ldots \ldots \ldots \ldots . \ldots \ldots$

6.4 Eigenvalues computed using eig, and using the method from Chapter 4, with $\alpha_{1}=4, \alpha_{2}=1$, and $\rho=3 / 8 \ldots \ldots \ldots \ldots$ 


\section{List of Illustrations}

1.1 Heat flow through composites of homogeneous materials . . . . . . . . . 1

3.1 Symbol of constant-coefficient operator [11] . . . . . . . . . . . . 7

3.2 Symbol of operator with piecewise constant coefficient [11] . . . . . . . . . 7

6.1 Solution with $\alpha_{1}=1, \alpha_{2}=2, \rho=3 / 8$ and $u(x, 0)=\sin (2 x) \ldots . . . .25$

6.2 Solution with $\alpha_{1}=1, \alpha_{2}=2, \rho=3 / 8$ and $u(x, 0)$ is a characteristic function . 26 


\title{
Chapter 1
}

\author{
Introduction
}

The purpose of this project is to model the diffusion of heat energy in one space dimension, such as within a rod, in the case where the heat flow is through a medium consisting of two or more homogeneous materials. For example, if the medium is a rod, the rod could be made of a copper rod and a silver rod welded together, as depicted in Figure 1.1. This figure is modeling the heat flow through an insulated rod made of two homogeneous materials, and the ends of the rod are immersed in mediums held at a fixed temperature at each end.

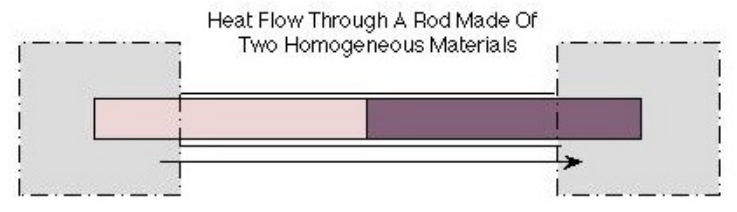

Figure 1.1: Heat flow through composites of homogeneous materials

The heat equation used to model the diffusion of heat through a single homogeneous material in a one-space dimension is as follows:

$$
u_{t}=\alpha^{2} u_{x x}
$$

where

$$
\begin{aligned}
u_{t} & =\text { rate of change in temperature with respect to time } \\
\alpha(x)^{2} & =\text { diffusivity } \\
u_{x x} & =\text { concavity of the temperature curve }
\end{aligned}
$$

The heat equation is a second order partial differential equation (PDE) that can be solved using a variety of analytical and numerical techniques. However, the challenge of creating a mathematical model for heat diffusion through a medium consisting of two or more homogeneous materials is that the heat equation will be modeled using a piecewise constant function $\alpha(x)^{2}$. The equation modeled then becomes the following equation:

$$
u_{t}=\left(\alpha(x)^{2} u_{x}\right)_{x}
$$


This occurs because the diffusivity changes based on the material. Unlike the heat equation with a constant coefficient, the heat equation with a discontinuous coefficients cannot be solved analytically. Normal methods of solving PDEs, such as separation of variables, cannot be used here. Numerical solution is difficult because discontinuous functions cannot be accurately represented using linear combinations of sines and cosines of varying frequencies, as is normally assumed when using numerical methods for such problems.

It is hypothesized that the solution can be represented as a linear combination of wave functions that change frequencies at the boundaries of different materials, like when heat is diffusing from one homogeneous material to another. The frequency changes are suggested by the Uncertainty Principle, which states that position and momentum of a particle can not be measured simultaneously with arbitrarily high precision. The Uncertainty Principle gives guidance on how to find eigenfunctions and eigenvalues of the differential operator $(\partial / \partial x) \alpha(x)^{2}(\partial / \partial x)$. With these approximate eigenfunctions, the solution can be represented in such a way that numerical solution of an equation with a discontinuous coefficient is almost as simple as that of an equation with a constant coefficient.

By solving such problems so accurately and efficiently, the proposed method will substantially aid researchers in science and engineering who need to simulate such heat diffusion processes. This will accelerate their workflow and provide new insight into the qualitative behavior of solutions. Although the proposed project only considers problems in one space dimension, such as modeling heat diffusion in a thin rod, the ideas in this project can be generalized to 2-D or 3-D models in future work. 


\section{Chapter 2}

Literature Review

The study of Partial Differential Equations (PDEs) is a fundamental part of mathematics because it links pure mathematics to applied mathematics by modeling physical phenomena such as fluid dynamics, magnetism, and heat flow. Most phenomena in these fields can be described using partial differential equations. A PDE is an equation that contains partial derivatives because the equation depends on multiple variables rather than depending on one variable like an Ordinary Differential Equation (ODE) [3]. The heat equation $u_{t}=\alpha(x)^{2} u_{x x}$, a second order PDE that depends on variable $t$ and $x$, will be studied. In this project, periodic boundary conditions are assumed so that the boundaries do not affect the solution; however, techniques from this project can be used on other boundary conditions, such as Dirichlet and Neumann boundary conditions. The heat equation models heat flow in one space dimension, and it can be solved simply with analytical or numerical methods when the diffusivity coefficient $\alpha(x)^{2}$ is a constant; however, when the heat equation is modeling heat diffusion through a medium consisting of two or more homogeneous materials, the heat equation will be modeled using a piecewise constant coefficient. This occurs because the diffusivity changes based on the material.

Why has the heat equation with a discontinuous coefficient not already been solved? One conventional way of solving PDEs is analytically. When solving a PDE with a constant coefficient analytically, the solution is represented in a series of sines and cosines because they are eigenfunctions of the spatial differential operator $\alpha^{2}(\partial / \partial x)$. The eigenfunctions of an operator have the property that when the operator is applied to the eigenfunction, it results in the eigenfunction times a number (eigenvalue), thus, the PDE becomes a simply solvable ODE. However, when the coefficients are not constant, this cannot be done because the eigenfunctions are unknown except in special cases such as the Lagrange, Bessel, or Hermite equations [1].

Problems arise when numerical methods are used to solve PDEs with discontinuous coefficients because the discontinuities in the coefficient $\alpha(x)^{2}$ lead to discontinuities in the solutions or its derivatives. Generally, the more continuous derivatives a function has, the more rapidly its Fourier series converges, but in this case, rapid convergence does not happen due to the discontinuities in the coefficient. As a result, solutions will have non-negligible high frequency components [9]. This results in the troublesome Gibbs phenomenon [9], which causes solutions to exhibit nonphysical oscillations. While there are methods such as 
those described in [6] for removing these oscillations, they are not practical to use in the context of a time-stepping method for solving a PDE. Any discontinuities will fade over time while progressing to a smooth steady-state solution, but until that happens, numerical methods will have difficulty due to stiffness. Stiffness occurs when solutions have both low- and high-frequency components that are coupled together and cannot be computed independently of one another, because they cannot be separated [2]. Because of stiffness, the highest-frequency component in the solution forces the time step used in numerical methods to be very small, even though the high-frequency components make a negligible contribution to the solution. This results in a dramatic increase in computational effort. This time step constraint is due to the CFL condition, which indicates how small the time step must be relative to the space step [9]. Since these issues arise in numerical methods, it is inconvenient and inefficient, and an alternative approach is needed.

It is hypothesized that these problems could be alleviated if approximate eigenfunctions of the spatial differential operator could be obtained in the case of a discontinuous coefficient. This could be done by representing the solution as a linear combination of wave functions that change frequencies at the boundaries of different materials, like when heat is diffusing from one homogeneous material to another.

A problem similar to the heat equation with a discontinuous coefficient, but with Dirichlet boundary conditions, was described and solved in [5]; however, they have no efficient method for computing eigenfunctions. For this problem, the $S A K$ principle derived from the Uncertainty Principle will be used to create a direct method for computing the eigenfunction of the spatial differential operator.

The frequency changes are suggested by the Uncertainty Principle, which states that position and momentum of a particle cannot be measured simultaneously with arbitrarily high precision. In mathematical terms, the Uncertainty Principle states that a function and its Fourier transform cannot be simultaneously concentrated in a arbitrarily small box in phase space [4], where phase space is the Cartesian product of physical space and frequency space. The Uncertainty Principle gives guidance on how to find eigenfunctions and eigenvalues of the differential operator $\alpha(x)^{2}(\partial / \partial x)$ [4] much more efficiently than standard methods from numerical linear algebra [8]. The eigenfunctions can be validated against those computed using numerical methods for computing eigenfunctions using matrices [8] which, while accurate, are too inefficient to use for the solution of PDE.

Initially, the approximate eigenfunctions were to be used in conjunction with Dr. Lambers' Krylov subspace spectral (KSS) methods, as described in [11], to design a numerical method for solving the heat equation that avoids the difficulties that existing methods have due to the discontinuous coefficient. KSS methods circumvent the difficulties associated 
with stiffness by computing each component of the solution, with respect to some chosen basis, using an approximation that is tailored specifically to that component [11]. To date, KSS methods have generally been used with a basis of sines and cosines, as they are approximate eigenfunctions for spatial differential operators with smoothly varying coefficients. We propose to instead use a basis of the approximate eigenfunctions described above, for the case of the discontinuous coefficient. For other PDEs, KSS methods have proven to be particularly effective solution methods when approximate (but not necessarily exact) eigenfunctions are available, thus making them an ideal choice compared to other time-stepping methods such as those described in [3, 9]. However, for the purpose of this project, instead of resorting to approximate eigenfunctions, the eigenfunctions can be computed as exact eigenfunctions, therefore, representing the solution as an eigenfunction expansion. 


\section{Chapter 3}

Methodology

To solve the heat equation

$$
u_{t}=\left(\alpha(x)^{2} u_{x}\right)_{x}
$$

with a discontinuous coefficient $\alpha(x)^{2}$, a numerical method will be designed using approximate eigenfunctions. It is hypothesized that the approximate eigenfunctions can be represented as a combination of wave functions that change frequency at different materials. The solution can be represented more efficiently as a combination of approximate eigenfunctions, rather than a combination of sines and cosines with fixed frequencies as is normally used for PDE with constant coefficients.

Before the approximate eigenfunctions can be constructed, the frequency changes at the interfaces between different homogeneous materials must be found. The Uncertainty Principle gives guidance on how to find the frequency changes. In Fefferman's article [4] on the Uncertainty Principle, he discusses the Uncertainty Principle in mathematical terms saying that a function $f(x)$ and its Fourier transform $\hat{f}(\omega)$ cannot be simultaneously concentrated in an arbitrarily small box in phase space, where phase space is the Cartesian product of physical space and frequency space. Fefferman builds on this with the $S A K$ principle, which states that given a differential operator $A(x, D)$, where $A(x, D)$ is self adjoint and positive definite so that eigenvalues are real and positive, and a number $K$, that the number of eigenvalues of $A(x, D)<K$, when $D=(1 / i) d / d x$ and $i=\sqrt{-1}$, is roughly equal to the number of distorted unit boxes that can be packed in the set of $S(A, K)$ where

$$
S(A, K)=\{(x, \omega) \mid A(x, \omega)<K\}
$$

and $A(x, \omega)$ is the symbol of the operator $A(x, D)$ According to the $S A K$ principle, the phase space can be divided into regions into which an eigenfunction can "fit" or can be mostly concentrated within. Furthermore, if a function $\phi(x)$ is mostly concentrated in phase space in a curved box, centered at $\left(x_{0}, \omega_{0}\right)$, then applying the operator $A(x, D)$ to this function yields

$$
A(x, D) \phi(x) \approx A\left(x_{0}, \omega_{0}\right) \phi(x)
$$

where $A\left(x_{0}, \omega_{0}\right)$ is an approximate eigenvalue, which means $\phi(x)$ is an approximate eigenfunction [4]. For this approximation to be more accurate, the symbol of the operator $A(x, \omega)$ should be nearly constant within the curved box. This suggests that we can find approximate 
eigenfunctions of the operator by studying the level curves of the symbol. The level curves jump at points of discontinuities, which suggest the changes in frequency. This phenomenon can be seen in Figure 3.1 and Figure 3.2 where the continuous frequency of the constant coefficient can be seen in Figure 3.1, and jumps in frequencies can be clearly seen in Figure 3.2 .

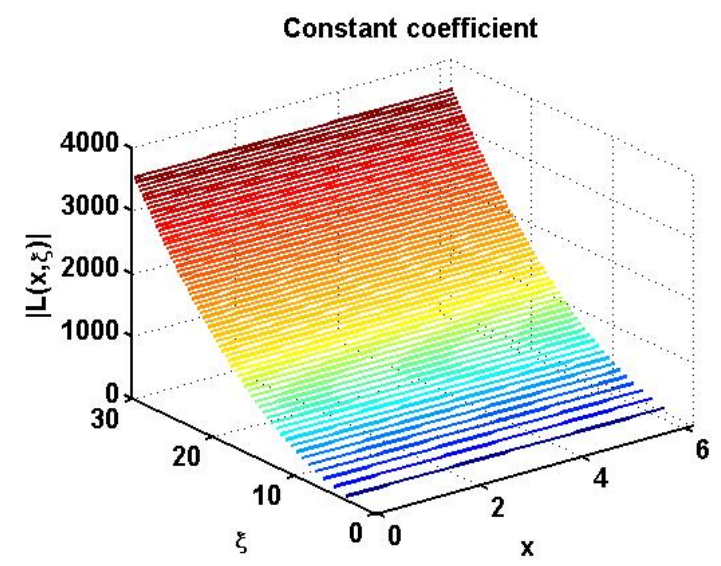

Figure 3.1: Symbol of constant-coefficient operator [11]

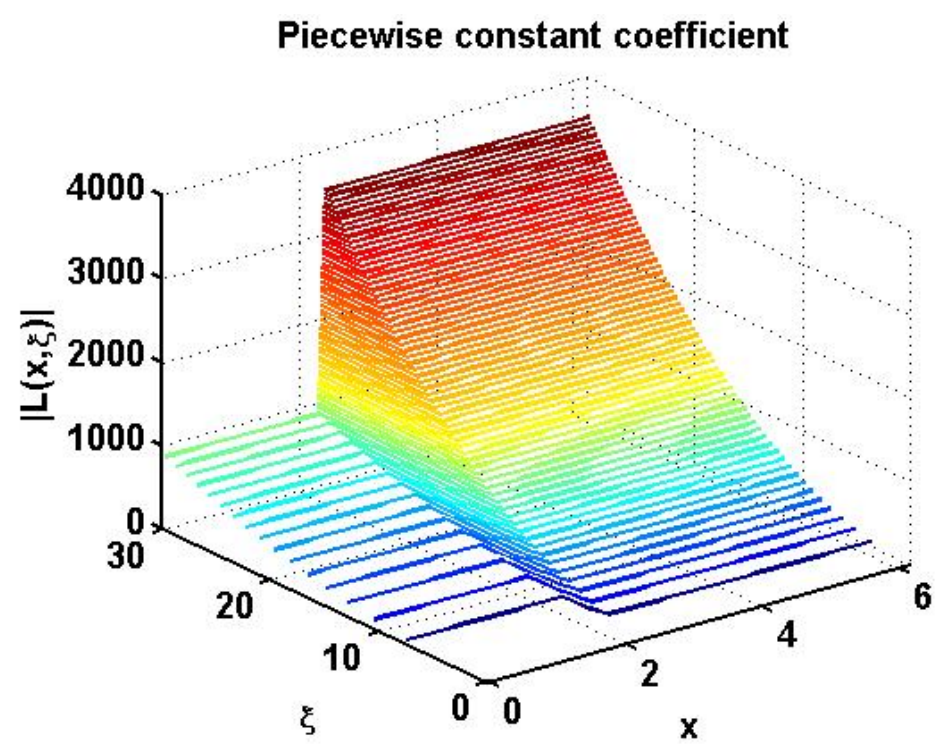

Figure 3.2: Symbol of operator with piecewise constant coefficient [11]

Furthermore, by using the $S A K$ principle, it is known that if

$$
\alpha(x)^{2}= \begin{cases}\alpha_{1}^{2} & 0<x<x_{0} \\ \alpha_{2}^{2} & x_{0}<x<2 \pi\end{cases}
$$


then $\alpha_{1}^{2} \omega_{1}^{2}=\alpha_{2}^{2} \omega_{2}^{2}$, where $\alpha_{k}$ is the diffusivity, $\omega_{k}$ is frequency, and $x_{0}$ is the point of discontinuity in the coefficient, and the volume of a region in phase space in which the eigenfunction is concentrated is $2 \pi$ [10].

Once the frequency changes are found, other unknown properties of the wave functions, including amplitude and phase shift, must still be computed, and then the approximate eigenfunctions can be constructed. For the spatial differential operator $\alpha(x)^{2}(\partial / \partial x)$, the smallest eigenfunction is zero; therefore, the first eigenfunction in the sequence is zero and the corresponding eigenfunction is a constant function.

The solution was to be represented as a linear combination using approximate eigenfunctions $\phi_{j}(x)$ and eigenvalues $\lambda_{j}$ for $j=1,2, \ldots$; however, in the next chapter, it is shown that the eigenfunctions can be computed very accurately, with most parameters determined analytically; therefore, the ideal eigenfunction expansion,

$$
u(x, t)=\sum_{n=1}^{\infty} \phi_{j}(x) e^{-\lambda_{j} t}\left\langle\phi_{j}, f(x)\right\rangle
$$

where the initial condition is

$$
u(x, 0)=f(x)
$$

and $f(x)$ represents the initial temperature profile of the rod can be used to represent the solution. In Chapter 5, it will also be shown how to obtain the coefficients of $f$ in the eigenfunction expansion from the coefficients $\hat{f}(\omega)$ of the discrete Fourier transform. If the eigenfunctions and eigenvalues were approximate, the fact that the approximate eigenfunctions are orthonormal can be used to represent the solution using the following formula: if we rewrite the heat equation in the form

$$
u_{t}+L u=0
$$

where

$$
L=-(\partial / \partial x) \alpha(x)^{2}(\partial / \partial x)
$$

and the solution is

$$
u(x, t)=e^{-L t} f(x)
$$

where the initial condition is

$$
u(x, 0)=f(x) .
$$

Then by expanding the above solution in the basis of eigenfunctions, the following formula is given for the solution:

$$
u(x, t)=\sum_{j=1}^{\infty} \phi_{j}(x)\left\langle\phi_{j}(x), e^{-L t} f\right\rangle
$$


where

$$
c_{j} \equiv\left\langle\phi_{j}(x), e^{-L t} f\right\rangle
$$

is a coefficient of the solution in basis $\left\{\phi_{j}\right\}$ and

$$
\langle f, g\rangle=\int_{0}^{2 \pi} f(x) g(x) d x
$$

which is the standard inner product on $(0,2 \pi)$.

This solution $u(x, t)$, however, cannot be computed numerically in this form; therefore, spatial discretization must be applied to the $c_{j}$ coefficent. Spatial discretization is used to transform a continuous partial differential equation into a system of discrete ordinary differential equations suitable for numerical computing. After discretization, the coefficient $c_{j}$ is approximated as follows:

$$
c_{j}=\left\langle\phi_{j}, e^{-L t} f\right\rangle \approx \vec{\phi}_{j}^{T} e^{-L_{N} t} \vec{f}
$$

where

$$
\vec{\phi}_{j}=\left[\begin{array}{c}
\phi_{j}\left(x_{0}\right) \\
\phi_{j}\left(x_{1}\right) \\
\vdots \\
\phi_{j}\left(x_{N-1}\right)
\end{array}\right]
$$

And similarly,

$$
\vec{f}=\left[\begin{array}{c}
f\left(x_{0}\right) \\
f\left(x_{1}\right) \\
\vdots \\
f\left(x_{N-1}\right)
\end{array}\right]
$$

where for both $\vec{\phi}_{j}$ and $\vec{f}$,

$$
x_{j}=j \Delta x
$$

and

$$
j=0,1, \ldots, N-1, \quad \Delta x=2 \pi / N .
$$

$L_{N}$ is an $N \times N$ matrix where

$$
L_{N}=-D_{N} A_{N}^{2} D_{N}
$$

where

$$
A_{N}=\left[\begin{array}{llllll}
\alpha & & & & & \\
& \ddots & & & & \\
& & \alpha_{1} & & & \\
& & & \alpha_{2} & & \\
& & & & \ddots & \\
& & & & & \alpha_{N}
\end{array}\right]
$$


and

$$
D_{N}=F_{N}^{-1} \Lambda_{N} F_{N}
$$

where $D_{N}$ represents the differentiation operator $d / d x$ and $L_{N}$ represents the differential operator $-(d / d x) \alpha(x)^{2}(d / d x)$. Then, $D_{N}$ will be represented as $F_{N}^{-1} \Lambda_{N} F_{N}$ where $F_{N}$ will be performing the discrete Fourier transform, and $\Lambda_{N}$ is a diagonal matrix containing eigenvalues of the differentiation operator [9]. The discrete Fourier transform can be used to compute the approximate Fourier series coefficients of the function. The formula for the discrete Fourier transform is as follows:

$$
f(x)=\frac{1}{2 \pi} \sum_{\omega=-\infty}^{\infty} e^{i \omega x} \hat{f}(\omega)
$$

and

$$
\hat{f}(\omega)=\frac{1}{2 \pi} \int_{0}^{2 \pi} e^{-i \omega x} f(x) d x
$$

where $\omega=-N / 2+1, \ldots, N / 2$.

After the coefficient $c_{j}$ is discretized, it is in matrix-vector form and can be approximated using techniques from "matrices, moments and quadrature" [7]. 


\section{Chapter 4}

Finding the Eigenfunction

We are modeling the solution as a linear combination of eigenfunctions that change frequency at the two different homogenous materials where an eigenfunction of an operator $A$ is a function $f$ such that the application of $A$ on $f$ gives the following equation:

$$
A f=\lambda f
$$

where $\lambda$ is called an eigenvalue and $f$ remains the same function. Since we are modeling the diffusion of heat energy through two homogeneous materials on the interval $[0,2 \pi]$, the eigenfunction that we are finding will have the following form:

$$
V_{j}(x)=\left\{\begin{array}{lr}
A_{j} \cos \left(\omega_{j 1} x-\theta_{j}\right) & 0 \leq x<2 \pi \rho \\
\cos \left(\omega_{j 2} x-\tau_{j}\right) & 2 \pi \rho \leq x<2 \pi
\end{array}\right.
$$

In the equation for the piecewise-defined eigenfunction, $V_{j 1}$ will represent the first piece on the interval $(0,2 \pi \rho)$ and $V_{j 2}$ will represent the second piece on the interval $(2 \pi \rho, 2 \pi)$ :

$$
\begin{gathered}
V_{j 1}(x)=A_{j} \cos \left(\omega_{j 1} x-\theta_{j}\right) \\
V j 2(x)=\cos \left(\omega_{j 2} x-\tau_{j}\right) .
\end{gathered}
$$

$2 \pi \rho$ represents the discontinuity point where $0<\rho<1$. The parameters $\theta_{j}$ and $\tau_{j}$ represent that phase shifts for the two homogenous materials; the parameters $\omega_{j 2}$ and $\omega_{j 1}$ represent the frequencies of the two homogenous materials; the parameter $A_{j}$ represents the amplitude for the first homogenous material. The eigenfunction will be normalized so that the amplitude for the second material is 1 for convenience.

The goal is to represent the solution as a linear combination of the eigenfunctions that change frequency where the heat energy changes from one homogeneous material to the other. To do this, all parameters except $\omega_{j 2}$ must be eliminated using the following conditions:

- $2 \pi$-periodicity continuity at the interface of the two homogeneous materials

- $S A K$ principle

- orthogonality 
- left-and right-hand conormal derivatives must be equal.

Once all parameters except $\omega_{j 2}$ have been eliminated, a numerical method, the secant method, will be used to approximate the frequency value $\omega_{j 2}$, using the $S A K$ principle to make initial guesses. A numerical method is necessary because eliminating the parameters results in a nonlinear equation for $\omega_{j 2}$.

The first parameter that will be eliminated is $A_{j}$. A formula for $A_{j} \cos (\theta)$ and $A_{j} \sin (\theta)$ must be found using the conditions of $2 \pi$-periodicity and continuity at the interface. $2 \pi$ Periodicity requires that the following relationship is true:

$$
V_{j}(2 \pi)=V_{j}(0)
$$

Then, since

$$
\begin{gathered}
V_{j}(0)=A_{j} \cos \left(\theta_{j}\right) \\
V_{j}(2 \pi)=\cos \left(\omega_{j 2} 2 \pi-\tau_{j}\right)
\end{gathered}
$$

then

$$
A_{j} \cos \left(\theta_{j}\right)=\cos \left(\omega_{j 2} 2 \pi-\tau_{j}\right) .
$$

Furthermore, the continuity at the interface stipulates

$$
A_{j} \cos \left(\omega_{j 1} 2 \pi \rho-\theta_{j}\right)=\cos \left(w_{j 2} 2 \pi \rho-\tau_{j}\right) .
$$

Then, by applying the trigonometric identity $\cos (\alpha-\beta)=\cos (\alpha) \cos (\beta)+\sin (\alpha) \sin (\beta)$ the new equation is

$$
A_{j} \cos \left(\omega_{j 1} 2 \pi \rho\right) \cos \left(\theta_{j}\right)+A_{j} \sin \left(\omega_{j 1} 2 \pi \rho\right) \sin \left(\theta_{j}\right)=\cos \left(w_{j 2} 2 \pi \rho-\tau_{j}\right) .
$$

Substituting in $A_{j} \cos \left(\theta_{j}\right)=\cos \left(\omega_{j 2} 2 \pi-\tau_{j}\right)$ the equation becomes

$$
\cos \left(\omega_{j 2} 2 \pi-\tau_{j}\right) \cos \left(\omega_{j 1} 2 \pi \rho\right)+A_{j} \sin \left(\omega_{j 1} 2 \pi \rho\right) \sin \left(\theta_{j}\right)=\cos \left(w_{j 2} 2 \pi \rho-\tau_{j}\right)
$$

and, thus,

$$
A_{j} \sin \left(\theta_{j}\right)=\frac{\cos \left(w_{j 2} 2 \pi \rho-\tau_{j}\right)-\cos \left(\omega_{j 1} 2 \pi \rho\right) \cos \left(\omega_{j 2} 2 \pi-\tau_{j}\right)}{\sin \left(\omega_{j 1} 2 \pi \rho\right)},
$$

assuming that $\sin \left(\omega_{j 1} 2 \pi \rho\right) \neq 0$.

By obtaining the formulas for $A_{j} \cos \left(\theta_{j}\right)$ and $A_{j} \sin \left(\theta_{j}\right)$, the parameters $\theta_{j}$ and $A_{j}$ can be effectively eliminated. After plugging in the formulas for $A_{j} \cos \left(\theta_{j}\right)$ and $A_{j} \sin \left(\theta_{j}\right)$, the two-material general eigenfunction has the form:

$$
V_{j}(x)=\left\{\begin{array}{lr}
\cos \left(\omega_{j 2} 2 \pi-\tau_{j}\right) \cos \left(\omega_{j 1} x\right)+ & \\
\frac{\cos \left(\omega_{j 2} 2 \pi \rho-\tau_{j}\right)-\cos \left(\omega_{j 1} 2 \pi \rho\right) \cos \left(\omega_{j 2} 2 \pi-\tau_{j}\right)}{\sin \left(\omega_{j 1} 2 \pi \rho\right)} \sin \left(\omega_{j 1} x\right) & 0 \leq x<2 \pi \rho \\
\cos \left(\omega_{j 2} x-\tau_{j}\right) & 2 \pi \rho \leq x<2 \pi
\end{array}\right.
$$


The next parameter that will be eliminated is $\omega_{j 1}$ using the $S A K$ principle, that states that $\alpha_{1} \omega_{j 1}=\alpha_{2} \omega_{j 2}$. Let $\phi=\frac{\alpha_{2}}{\alpha_{1}}$ so that $\omega_{j 1}=\phi \omega_{j 2}$. Substituting in the equation for $\omega_{j 1}$, the equation for our eigenfunction is now in this form:

$$
V_{j}(x)=\left\{\begin{array}{lr}
\cos \left(\omega_{j 2} 2 \pi-\tau_{j}\right) \cos \left(\phi \omega_{j 2} x\right)+ & \\
\frac{\cos \left(\omega_{j 2} 2 \pi \rho-\tau_{j}\right)-\cos \left(\phi \omega_{j 2} 2 \pi \rho\right) \cos \left(\omega_{j 2} 2 \pi-\tau_{j}\right)}{\sin \left(\phi \omega_{j 2} 2 \pi \rho\right)} \sin \left(\phi \omega_{j 2} x\right) & 0 \leq x<2 \pi \rho \\
\cos \left(\omega_{j 2} x-\tau_{j}\right) & 2 \pi \rho \leq x<2 \pi
\end{array} .\right.
$$

Thirdly, $\tau_{j}$ is eliminated by using the condition that the left- and right-hand conormal derivatives must be equal and the condition of orthogonality. First using the left- and righthand derivatives, it is known that if the left- and right-hand derivatives are scaled by the $\alpha_{1}^{2}$ and $\alpha_{2}^{2}$, respectively, then their slopes must be equal. To do this, $V_{j}(x)$ is separated into its two pieces, $V_{j 1}(x)$ and $V_{j 2}(x)$, and the derivative of both pieces is taken.

$$
\begin{aligned}
V_{j 1}^{\prime}(x)= & -\phi \omega_{j 2} \cos \left(\omega_{j 2} 2 \pi-\tau_{j}\right) \sin \left(\phi \omega_{j 2} x\right)+ \\
& \frac{\cos \left(\omega_{j 2} 2 \pi \rho-\tau_{j}\right)-\cos \left(\phi \omega_{j 2} 2 \pi \rho\right) \cos \left(\omega_{j 2} 2 \pi-\tau_{j}\right)}{\sin \left(\phi \omega_{j 2} 2 \pi \rho\right)} \phi \omega_{j 2} \cos \left(\phi \omega_{j 2} x\right) \\
V_{j 2}^{\prime}(x)= & -\omega_{j 2} \sin \left(\omega_{j 2} x-\tau_{j}\right) .
\end{aligned}
$$

The conomoral condition specifies that the eigenfunction must satisfy the following equation:

$$
\alpha_{1}^{2} V_{j 1}^{\prime}(2 \pi \rho)=\alpha_{2}^{2} V_{j 2}^{\prime}(2 \pi \rho)
$$

Therefore, plugging in $x=2 \pi \rho$ in both derivatives yields

$$
\begin{aligned}
V_{j 1}^{\prime}(2 \pi \rho)= & -\phi \omega_{j 2} \cos \left(\omega_{j 2} 2 \pi-\tau_{j}\right) \sin \left(\phi \omega_{j 2} 2 \pi \rho\right)+ \\
& \frac{\cos \left(\omega_{j 2} 2 \pi \rho-\tau_{j}\right)-\cos \left(\phi \omega_{j 2} 2 \pi \rho\right) \cos \left(\omega_{j 2} 2 \pi-\tau_{j}\right)}{\sin \left(\phi \omega_{j 2} 2 \pi \rho\right)} \phi \omega_{j 2} \cos \left(\phi \omega_{j 2} 2 \pi \rho\right) \\
V_{j 2}^{\prime}(2 \pi \rho)= & -\omega_{j 2} \sin \left(\omega_{j 2} 2 \pi \rho-\tau_{j}\right) .
\end{aligned}
$$

Using the condition, we obtain $\alpha_{1}^{2} V_{j 1}^{\prime}(2 \pi \rho)=\alpha_{2}^{2} V_{j 2}^{\prime}(2 \pi \rho)$

$$
\begin{aligned}
\alpha_{2}^{2} \omega_{j 2} \sin \left(\omega_{j 2} 2 \pi \rho-\tau_{j}\right)= & \alpha_{1}^{2}-\phi \omega_{j 2} \cos \left(\omega_{j 2} 2 \pi-\tau_{j}\right) \sin \left(\phi \omega_{j 2} 2 \pi \rho\right)+ \\
& \frac{\cos \left(\omega_{j 2} 2 \pi \rho-\tau_{j}\right)-\cos \left(\phi \omega_{j 2} 2 \pi \rho\right) \cos \left(\omega_{j 2} 2 \pi-\tau_{j}\right)}{\sin \left(\phi \omega_{j 2} 2 \pi \rho\right)} \times \\
& \phi \omega j 2 \cos \left(\phi \omega_{j 2} 2 \pi \rho\right) .
\end{aligned}
$$

Using $\phi=\frac{\alpha_{2}}{\alpha_{1}}$ and simplifying, the equation reduces to:

$$
\begin{aligned}
\phi \sin \left(\omega_{j 2} 2 \pi \rho-\tau_{j}\right)= & -\cos \left(\omega_{j 2} 2 \pi-\tau_{j}\right) \sin \left(\phi \omega_{j 2} 2 \pi \rho\right)+ \\
& \frac{\cos \left(\omega_{j 2} 2 \pi \rho-\tau_{j}\right)-\cos \left(\phi \omega_{j 2} 2 \pi \rho\right) \cos \left(\omega_{j 2} 2 \pi-\tau_{j}\right)}{\sin \left(\phi \omega_{j 2} 2 \pi \rho\right)} \times \\
& \cos \left(\phi \omega_{j 2} 2 \pi \rho\right) .
\end{aligned}
$$


Next, the trigonometric functions should be expanded using trigonometric identities so that $\tau_{j}$ can be isolated.

$$
\begin{aligned}
-\phi \sin \left(\omega_{j 2} 2 \pi \rho\right) \cos \left(\tau_{j}\right)+ & \\
\phi \cos \left(\omega_{j 2} 2 \pi \rho\right) \sin \left(\tau_{j}\right)= & -\sin \left(\phi \omega_{j 2} 2 \pi \rho\right)\left[\cos \left(\omega_{j 2} 2 \pi\right) \cos \left(\tau_{j}\right)+\sin \left(\omega_{j 2} 2 \pi\right) \sin \left(\tau_{j}\right)\right]+ \\
& \frac{\cos \left(\phi \omega_{j 2} 2 \pi \rho\right)}{\sin \left(\phi \omega_{j 2} 2 \pi \rho\right)}\left[\cos \left(\omega_{j 2} 2 \pi \rho\right) \cos \left(\tau_{j}\right)+\sin \left(\omega_{j 2} 2 \pi \rho\right) \sin \left(\tau_{j}\right)\right]- \\
& \frac{\cos ^{2}\left(\phi \omega_{j 2} 2 \pi \rho\right)}{\sin \left(\phi \omega_{j 2} 2 \pi \rho\right)}\left[\cos \left(\omega_{j 2} 2 \pi\right) \cos \left(\tau_{j}\right)+\sin \left(\omega_{j 2} 2 \pi\right) \sin \left(\tau_{j}\right)\right]
\end{aligned}
$$

Now $\cos \left(\tau_{j}\right)$ and $\sin \left(\tau_{j}\right)$ can be separated in the equation.

$$
\begin{aligned}
0= & \cos \left(\tau_{j}\right)\left[-\cos \left(\omega_{j 2} 2 \pi\right)+\cos \left(\phi \omega_{j 2} 2 \pi \rho\right) \cos \left(\omega_{j 2} 2 \pi \rho\right)+\phi \sin \left(\omega_{j 2} 2 \pi \rho\right) \sin \left(\phi \omega_{j 2} 2 \pi \rho\right)\right]+ \\
& \sin \left(\tau_{j}\right)\left[-\sin \left(\omega_{j 2} 2 \pi\right)+\cos \left(\phi \omega_{j 2} 2 \pi \rho\right) \sin \left(\omega_{j 2} 2 \pi \rho\right)-\phi \cos \left(\omega_{j 2} 2 \pi \rho\right) \sin \left(\phi \omega_{j 2} 2 \pi \rho\right)\right]
\end{aligned}
$$

Finally, the eigenfunction needs to be integrated to be sure that it satisfies the orthogonality condition

$$
<V_{j}, V_{0}>=\int_{0}^{2 \pi} V_{j}(x) V_{0} d x=0
$$

where if $V_{0}$ is the eigenfunction that corresponds to the smallest eigenvalue $\lambda_{0}=0$, then $V_{0}=1$; therefore, the equation from the orthogonality condition becomes $\int_{0}^{2 \pi} V_{j}(x) d x$. Plugging in $V_{j}(x)$ in the integral, the following needs to be evaluated:

$$
\begin{aligned}
& \int_{0}^{2 \pi} \frac{\cos \left(\omega_{j 2} 2 \pi \rho-\tau_{j}\right)-\cos \left(\phi \omega_{j 2} 2 \pi \rho\right) \cos \left(\omega_{j 2} 2 \pi-\tau_{j}\right)}{\sin \left(\phi \omega_{j 2} 2 \pi \rho\right)} \sin \left(\phi \omega_{j 2} x\right)+ \\
& \cos \left(\omega_{j 2} 2 \pi-\tau_{j}\right) \cos \left(\phi \omega_{j 2} x\right)+\cos \left(\omega_{j 2} x-\tau_{j}\right) d x=0 .
\end{aligned}
$$

Because the eigenfunction is a piecewise-defined eigenfunction with the discontinuity point $2 \pi \rho$, the orthogonality condition can be written as

$$
\int_{0}^{2 \pi \rho} V_{j 1}(x) d x+\int_{2 \pi \rho}^{2 \pi} V_{j 2}(x) d x
$$

which is equivalent to

$$
\begin{gathered}
\int_{0}^{2 \pi \rho} \cos \left(\omega_{j 2} 2 \pi-\tau_{j}\right) \cos \left(\phi \omega_{j 2} x\right)+ \\
\frac{\cos \left(\omega_{j 2} 2 \pi \rho-\tau_{j}\right)-\cos \left(\phi \omega_{j 2} 2 \pi \rho\right) \cos \left(\omega_{j 2} 2 \pi-\tau_{j}\right)}{\sin \left(\phi \omega_{j 2} 2 \pi \rho\right)} \sin \left(\phi \omega_{j 2} x\right) d x+ \\
\int_{2 \pi \rho}^{2 \pi} \cos \left(\omega_{j 2} x-\tau_{j}\right) d x=0 .
\end{gathered}
$$


Then, the integral can be evaluated as follows:

$$
\begin{aligned}
0= & \cos \left(\omega_{j 2} 2 \pi-\tau_{j}\right) \sin ^{2}\left(\phi \omega_{j 2} 2 \pi \rho\right)-\cos \left(\omega_{j 2} 2 \pi \rho-\tau_{j}\right) \cos \left(\phi \omega_{j 2} 2 \pi \rho\right)+ \\
& \cos \left(\omega_{j 2} 2 \pi-\tau_{j}\right) \cos ^{2}\left(\phi \omega_{j 2} 2 \pi \rho\right)+\phi \cos \left(\omega_{j 2} 2 \pi \rho-\tau_{j}\right)- \\
& \phi \cos \left(\phi \omega_{j 2} 2 \pi \rho\right) \cos \left(\omega_{j 2} 2 \pi-\tau_{j}\right)+\phi \sin \left(\phi \omega_{j 2} 2 \pi \rho\right)\left[\sin \left(\omega_{j 2} 2 \pi\right) \cos \left(\tau_{j}\right)-\right. \\
& \left.\cos \left(\omega_{j 2} 2 \pi\right) \sin \left(\tau_{j}\right)-\sin \left(\omega_{j 2} 2 \pi \rho\right) \cos \left(\tau_{j}\right)+\cos \left(\omega_{j 2} 2 \pi \rho\right) \sin \left(\tau_{j}\right)\right]
\end{aligned}
$$

Next, the evaluated integral should be $\operatorname{simplified~and~the~} \cos \left(\tau_{j}\right)$ and $\sin \left(\tau_{j}\right)$ should be grouped together.

$$
\begin{aligned}
0= & \cos \left(\tau_{j}\right)\left[\cos \left(\omega_{j 2} 2 \pi\right)-\cos \left(\omega_{j 2} 2 \pi \rho\right) \cos \left(\phi \omega_{j 2} 2 \pi \rho\right)+\cos \left(\omega_{j 2} 2 \pi \rho\right)-\right. \\
& \left.\left.\cos \left(\phi \omega_{j 2} 2 \pi \rho\right) \cos \left(\omega_{j 2} 2 \pi \rho\right)+\phi \sin \left(\omega_{j 2} 2 \pi \rho\right)\left(\sin \left(\omega_{j 2} 2 \pi\right)-\sin \left(\omega_{j 2} 2 \pi \rho\right)\right)\right] 4.4\right) \\
& +\sin \left(\tau_{j}\right)\left[\sin \left(\omega_{j 2} 2 \pi\right)-\sin \left(\omega_{j 2} 2 \pi \rho\right) \cos \left(\phi \omega_{j 2} 2 \pi \rho\right)+\sin \left(\omega_{j 2} 2 \pi \rho\right)-\right. \\
& \left.\cos \left(\phi \omega_{j 2} 2 \pi \rho\right) \sin \left(\omega_{j 2} 2 \pi \rho\right)-\phi \sin \left(\omega_{j 2} 2 \pi \rho\right)\left(\cos \left(\omega_{j 2} 2 \pi\right)-\cos \left(\omega_{j 2} 2 \pi \rho\right)\right)\right]
\end{aligned}
$$

Now $\tau_{j}$ must be eliminated by taking equation (4.1) and (4.2) and making a homogeneous system of two equations in two unknowns, $\cos \left(\tau_{j}\right)$ and $\sin \left(\tau_{j}\right)$. Doing this, we introduce the following four equations where $G_{1}$ and $G_{2}$ are coefficients of $\cos \left(\tau_{j}\right)$ and $\sin \left(\tau_{j}\right)$, respectively, from the orthogonality condition, and $H_{1}$ and $H_{2}$ are the coefficients for the matching of conormal derivatives. :

$$
\begin{aligned}
G_{1}= & \cos \left(\omega_{j 2} 2 \pi\right)-\cos \left(\omega_{j 2} 2 \pi \rho\right) \cos \left(\phi \omega_{j 2} 2 \pi \rho\right)+\cos \left(\omega_{j 2} 2 \pi \rho\right)- \\
& \cos \left(\phi \omega_{j 2} 2 \pi \rho\right) \cos \left(\omega_{j 2} 2 \pi \rho\right)+\phi \sin \left(\omega_{j 2} 2 \pi \rho\right)\left(\sin \left(\omega_{j 2} 2 \pi\right)-\sin \left(\omega_{j 2} 2 \pi \rho\right)\right) \\
G_{2}= & \sin \left(\omega_{j 2} 2 \pi\right)-\sin \left(\omega_{j 2} 2 \pi \rho\right) \cos \left(\phi \omega_{j 2} 2 \pi \rho\right)+\sin \left(\omega_{j 2} 2 \pi \rho\right)- \\
& \cos \left(\phi \omega_{j 2} 2 \pi \rho\right) \sin \left(\omega_{j 2} 2 \pi \rho\right)-\phi \sin \left(\omega_{j 2} 2 \pi \rho\right)\left(\cos \left(\omega_{j 2} 2 \pi\right)-\cos \left(\omega_{j 2} 2 \pi \rho\right)\right) \\
H_{1}=- & \cos \left(\omega_{j 2} 2 \pi\right)+\cos \left(\phi \omega_{j 2} 2 \pi \rho\right) \cos \left(\omega_{j 2} 2 \pi \rho\right)+\phi \sin \left(\omega_{j 2} 2 \pi \rho\right) \sin \left(\phi \omega_{j 2} 2 \pi \rho\right) \\
H_{2}=- & \sin \left(\omega_{j 2} 2 \pi\right)+\cos \left(\phi \omega_{j 2} 2 \pi \rho\right) \sin \left(\omega_{j 2} 2 \pi \rho\right)-\phi \cos \left(\omega_{j 2} 2 \pi \rho\right) \sin \left(\phi \omega_{j 2} 2 \pi \rho\right)
\end{aligned}
$$

We use these four expressions to create the homogeneous system.

$$
\left[\begin{array}{ll}
G_{1} & G_{2} \\
H_{1} & H_{2}
\end{array}\right]\left[\begin{array}{c}
\cos \left(\tau_{j}\right) \\
\sin \left(\tau_{j}\right)
\end{array}\right]=\left[\begin{array}{l}
0 \\
0
\end{array}\right]
$$

In order for this system to have a nontrivial solution, the determinant of the matrix must be zero; therefore, the equation $G_{1} H_{2}-G_{2} H_{1}=0$ is entered into MATLAB and iterated using 
the secant method to solve for $\omega_{j 2}$. The secant method is used because it has a rapid rate of convergence, but unlike Newton's method, which converges more rapidly, it does not require evaluating a derivative which would be very complex for the determinant.

The initial guesses for the secant method are found by using the $S A K$ principle that states that the volume of the region into which eigenfunction is concentrated in phase space must be $2 \pi$.

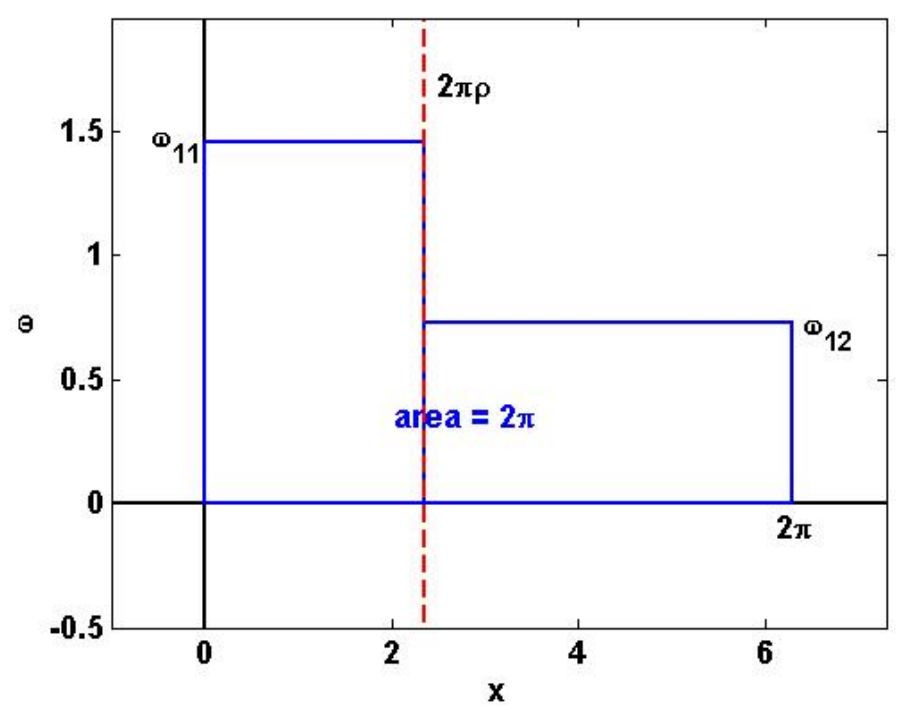

Since we know that the area $=2 \pi$ and as an example for this project, $\rho=\frac{3}{8}$ and the point of discontinuity $x_{0}=2 \pi \rho$, the following equation must be true:

$$
2 \pi=\omega_{j 1} \frac{3 \pi}{4}+\omega_{j 2}\left(2 \pi-\frac{3 \pi}{4}\right)
$$

Based on the $S A K$ principle that states $\alpha_{1} \omega_{j 1}=\alpha_{2} \omega_{j 2}$, the equation simplifies to

$$
\begin{gathered}
2 \pi=2 \omega_{j 2} \frac{3 \pi}{4}+\omega_{j 2} \frac{5 \pi}{4} \\
\omega_{j 2}=\frac{8}{11} .
\end{gathered}
$$

Therefore, $\frac{8}{11}$ and multiples of $\frac{8}{11}$ are used as initial guesses to compute approximate frequency values for the eigenfunctions. It has previously been stated that for $A_{j} \sin \left(\theta_{j}\right)$ to have a real defined value $\sin \left(\omega_{j 1} 2 \pi \rho\right) \neq 0$; however, we want to investigate whether it is still possible to obtain eigenfunctions when $\sin \left(\omega_{j 1} 2 \pi \rho\right)=0$. Therefore, to do this, we let $\omega_{j 1}=\frac{k}{2 \rho}$ where $k$ is an integer. Then the new formula for $\omega_{j 1}$ must be substituted into $V_{j}$ and then applied to the conditions periodicity, continuity, orthogonality, SAK, and conormal 
derivatives so that we have

$$
V_{j}(x)=\left\{\begin{array}{lr}
A_{j} \cos \left(\frac{k}{2 \rho} x-\theta_{j}\right) & 0 \leq x<2 \pi \rho \\
\cos \left(\frac{k}{\phi 2 \rho} x-\tau_{j}\right) & 2 \pi \rho \leq x<2 \pi
\end{array}\right.
$$

After the $\omega_{j 1}$ substitution, the periodicity condition is as follows:

$$
\begin{gathered}
V_{j}(0)=A_{j} \cos \left(\theta_{j}\right) \\
V_{j}(2 \pi)=\cos \left(\frac{k}{\phi 2 \rho} 2 \pi-\tau_{j}\right)
\end{gathered}
$$

then

$$
A_{j} \cos \left(\theta_{j}\right)=\cos \left(\frac{k}{\phi 2 \rho} 2 \pi-\tau_{j}\right)
$$

With the substitution, the continuity condition is as follows:

$$
\begin{gathered}
A_{j} \cos \left(\frac{k}{2 \rho} 2 \pi \rho-\theta_{j}\right)=\cos \left(\frac{k}{\phi 2 \rho} 2 \pi \rho-\tau_{j}\right) \\
A_{j} \cos \left(\frac{k}{2 \rho} 2 \pi \rho\right) \cos \left(\theta_{j}\right)+A_{j} \sin \left(\frac{k}{w \rho}\right) \sin \left(\theta_{j}\right)=\cos \left(\frac{k}{\phi 2 \rho} 2 \pi \rho-\tau_{j}\right) \\
\cos (k \pi) \cos \left(\frac{k}{\phi 2 \rho} 2 \pi-\tau_{j}\right)+A_{j} \sin (k \pi) \sin \left(\theta_{j}\right)=\cos \left(\frac{k}{\phi 2 \rho} 2 \pi \rho-\tau_{j}\right) \\
(-1)^{k} \cos \left(\frac{k}{\phi 2 \rho} 2 \pi-\tau_{j}\right)=\cos \left(\frac{k}{\phi 2 \rho} 2 \pi \rho-\tau_{j}\right) \\
(-1)^{k} \cos \left(\frac{k \pi}{\phi \rho}-\tau_{j}\right)=\cos \left(\frac{k \pi}{\phi}-\tau_{j}\right)
\end{gathered}
$$

Then taking the orthogonality condition that states

$$
\int_{0}^{2 \pi \rho} A_{j} \cos \left(\omega_{j 1} x-\theta_{j}\right) d x+\int_{2 \pi \rho}^{2 \pi} \cos \left(\frac{k}{\omega_{j 2}} x-\tau_{j}\right) d x=0
$$

and then plugging in the new condition, we have the following:

$$
\int_{0}^{2 \pi \rho} A_{j} \cos \left(\frac{k}{2 \rho} x-\theta_{j}\right) d x+\int_{2 \pi \rho}^{2 \pi} \cos \left(\frac{k}{\phi 2 \rho} x-\tau_{j}\right) d x=0 .
$$

Then the integral can be evaluated:

$$
\frac{A_{j} 2 \rho}{k}\left[\sin \left(k \pi-\theta_{j}\right)+\sin \left(\theta_{j}\right)\right]+\frac{\phi 2 \rho}{k}\left[\sin \left(\frac{k \pi}{\phi \rho}-\tau_{j}\right)-\sin \left(\frac{k \pi}{\phi}-\tau_{j}\right)\right]
$$

Then this expression should be solved for $A_{j} \sin \left(\theta_{j}\right)$ :

$$
A_{j} \sin \left(\theta_{j}\right)=\frac{\phi \sin \left(\frac{k \pi}{\phi \rho}-\tau_{j}\right)-\phi \sin \left(\frac{k \pi}{\phi}-\tau_{j}\right)}{1-(-1)^{k}} .
$$


The property of equality of right- and left-hand conormal derivatives that states

$$
\alpha_{1}^{2} V_{j 1}^{\prime}(2 \pi \rho)=\alpha_{2}^{2} V_{j 2}^{\prime}(2 \pi \rho)
$$

Evaluating this expression, we get the following expression:

$$
-A_{j} \frac{k}{2 \rho} \alpha_{1}^{2} \sin \left(k \pi-\theta_{j}\right)=-\frac{k}{2 \rho} \alpha_{2}^{2} \sin \left(\frac{k \pi}{\phi}-\tau_{j}\right) .
$$

Next, rearrange the expression to get an expression for $A_{j} \sin \left(\theta_{j}\right)$.

$$
A_{j} \sin \left(\theta_{j}\right)=-(-1)^{k} \phi \sin \left(\frac{k \pi}{\phi}-\tau_{j}\right)
$$

Then use Equation 4.7 equal Equation 4.8 to get the following equation:

$$
(-1)^{k} \sin \left(\frac{k \pi}{\phi \rho}-\tau_{j}\right)=\sin \left(\frac{k \pi}{\phi}-\tau_{j}\right)
$$

Equation 4.6 from the continuity condition and Equation 4.9 can be divided used to find the condition for $\omega_{j 1}$.

$$
\frac{\sin \left(\frac{k \pi}{\phi}-\tau_{j}\right)}{\cos \left(\frac{k \pi}{\phi}-\tau_{j}\right)}=\frac{(-1)^{k} \sin \left(\frac{k \pi}{\phi \rho}-\tau_{j}\right)}{(-1)^{k} \cos \left(\frac{k \pi}{\phi \rho}-\tau_{j}\right)}
$$

Using trigonometric identities, the equation can be simplified to the following:

$$
\tan \left(\frac{k \pi}{\phi}-\tau_{j}\right)=\tan \left(\frac{k \pi}{\phi \rho}-\tau_{j}\right) .
$$

We can take this equation and solve for the conditions that must be imposed on $n$ to make the eigenfunction defined.

$$
\begin{gathered}
\frac{k \pi}{\phi}=\frac{k \pi}{\phi \rho}+n \pi \\
k-n \phi=\frac{k}{\rho}
\end{gathered}
$$

This equation gives us the the following equations for $\rho$ and $n$ :

$$
\begin{gathered}
\rho=\frac{k}{k-n \phi} \\
n=\frac{k}{\phi}\left(1-\frac{1}{\rho}\right)
\end{gathered}
$$

where we know that $\rho$ must be on the interval $(0,1)$ because $2 \pi \rho$ must be on the interval $(0,2 \pi)$, thus, we know that $n \nsupseteq 0$ because if $n \geq 0, \rho<0$ or $\rho \geq 1$. Therefore, $n<0$ is a necessary condition for an eigenfunction to exist. 


\section{Chapter 5}

Solving the PDE

Once the approximate eigenfunctions are constructed, the PDE can be solved. Modeling the $2 \pi$-periodic heat equation gives the following PDE:

$$
\frac{\partial u}{\partial t}=\frac{\partial}{\partial x}\left(\alpha(x)^{2} \frac{\partial u}{\partial x}\right) \quad 0<x<2 \pi
$$

where

$$
u(x, 0)=f(x)
$$

This initial-boundary problem has a solution of the form:

$$
u(x, t)=\sum_{j=0}^{\infty} u_{j} e^{\lambda_{j} t} V_{j}(x)
$$

where

$$
\lambda_{j}=-\alpha_{2}^{2} \omega_{j 2}^{2}
$$

and

$$
u_{j}=\frac{\int_{0}^{2 \pi} V_{j}(x) f(x) d x}{\int_{0}^{2 \pi} V_{j}(x)^{2} d x}
$$

The denominator can be evaluated analytically:

$$
\begin{aligned}
\int_{0}^{2 \pi} V_{j}(x)^{2} d x= & \int_{0}^{2 \pi \rho} A_{j}^{2} \cos ^{2}\left(\omega_{j 1} x-\theta_{j}\right) d x+\int_{2 \pi \rho}^{2 \pi} \cos ^{2}\left(\omega_{j 2} x-\tau_{j}\right) d x \\
= & A_{j}^{2} \int_{0}^{2 \pi \rho} \frac{1}{2}\left(1+\cos \left(2 \omega_{j 1} x-2 \theta_{j}\right)\right) d x \\
& +\int_{2 \pi \rho}^{2 \pi} \frac{1}{2}\left(1+\cos \left(2 \omega_{j 2} x-2 \tau_{j}\right)\right) d x \\
= & \left.\frac{A_{j}^{2}}{2}\left[x+\frac{\sin \left(2 \omega_{j 1} x-2 \theta_{j}\right)}{2 \omega_{j 1}}\right]\right|_{0} ^{2 \pi \rho}+\left.\left[\frac{x}{2}+\frac{\sin \left(2 \omega_{j 2} x-2 \tau_{j}\right)}{4 \omega_{j 2}}\right]\right|_{2 \pi \rho} ^{2 \pi} \\
= & \frac{A_{j}^{2}}{2}\left(\left[2 \pi \rho+\frac{\sin \left(4 \omega_{j 1} \pi \rho-2 \theta_{j}\right)}{2 \omega_{j 1}}\right]-\left[\frac{\sin \left(-2 \theta_{j}\right)}{2 \omega_{j 1}}\right]\right) \\
& +\left(\left[\pi+\frac{\sin \left(4 \omega_{j 2} \pi-2 \tau_{j}\right)}{4 \omega_{j 2}}\right]-\left[\pi \rho+\frac{\sin \left(4 \omega_{j 2} \pi \rho-2 \tau_{j}\right)}{2 \omega_{j 2}}\right]\right)
\end{aligned}
$$

The numerator can be represented with a Fourier series and computed numerically. 
Let

$$
u_{j}=\frac{\int_{0}^{2 \pi} V_{j}(x) f(x) d x}{\int_{0}^{2 \pi} V_{j}(x)^{2} d x}=\frac{\left\langle V_{j}(x), f(x)\right\rangle}{c_{j}}
$$

where $\left\langle V_{j}(x), f(x)\right\rangle$ refers to the inner product like (3.1) and $f(x)$ has the following Fourier series:

$$
f(x)=\frac{a_{0}}{2}+\sum_{k=1}^{\infty} a_{k} \cos (k x)+b_{k} \sin (k x)
$$

Therefore,

$$
\left\langle V_{j}(x), f(x)\right\rangle=\frac{a_{0}}{2}\left\langle V_{j}, 1\right\rangle+\sum_{k=1}^{N} a_{k}\left\langle V_{j}, \cos (k x)\right\rangle+b_{k}\left\langle V_{j}, \sin (k x)\right\rangle
$$

The Fourier transform of the given function can be computed numerically using fast Fourier transform to find $a_{k}, b_{k}$, and $a_{0}$. Then the $\omega_{j 2}$ frequency values can be found by solving the determinant $G_{1} H_{2}-G_{2} H_{1}=0$ for $\omega_{j 2}$ discussed in Chapter 4. Once the values for $\omega_{j 2}$ are known, the eigenfunction can be reconstructed using the formulas for $A_{j}, \cos \left(\theta_{j}\right)$, $\sin \left(\theta_{j}\right), \cos \left(\tau_{j}\right), \sin \left(\tau_{j}\right)$, and $\omega_{j 1} . \omega_{j 1}$ can be represented similarly as before, using the SAK principle that states $\omega_{j 1}=\frac{\alpha_{2}}{\alpha_{1}} \omega_{j 2} . A_{j}$ and $\theta_{j}$ can be found using the formulas for $A_{j} \sin \left(\theta_{j}\right)$ and $A_{j} \cos \left(\theta_{j}\right)$ :

$$
A_{j} \sin \left(\theta_{j}\right)=\frac{\cos \left(w_{j 2} 2 \pi \rho-\tau_{j}\right)-\cos \left(\omega_{j 1} 2 \pi \rho\right) \cos \left(\omega_{j 2} 2 \pi-\tau_{j}\right)}{\sin \left(\omega_{j 1} 2 \pi \rho\right)}
$$

and

$$
A_{j} \cos \left(\theta_{j}\right)=\cos \left(\omega_{j 2} 2 \pi-\tau_{j}\right)
$$

Manipulating these formulas, we have the following equation for $A_{j}$ :

$$
A_{j}=\sqrt{\left(\cos \left(\omega_{j 2} 2 \pi-\tau_{j}\right)\right)^{2}+\left(\frac{\cos \left(\omega_{j 2} 2 \pi \rho-\tau_{j}\right)-\cos \left(\omega_{j 1} 2 \pi \rho\right) \cos \left(\omega_{j 2} 2 \pi-\tau_{j}\right)}{\sin \left(\omega_{j 1} 2 \pi \rho\right)}\right)^{2}}
$$

Then using the identity $\tan \left(\theta_{j}\right)=\frac{\sin \left(\theta_{j}\right)}{\cos \left(\theta_{j}\right)}$ and applying it to the formulas $A_{j} \sin \left(\theta_{j}\right)$ and $A_{j} \cos \left(\theta_{j}\right)$, we generate the following formula for $\tan \left(\theta_{j}\right)$ :

$$
\tan \left(\theta_{j}\right)=\frac{\cos \left(w_{j 2} 2 \pi \rho-\tau_{j}\right)-\cos \left(\omega_{j 1} 2 \pi \rho\right) \cos \left(\omega_{j 2} 2 \pi-\tau_{j}\right)}{\sin \left(\omega_{j 1} 2 \pi \rho\right) \cos \left(\omega_{j 2} 2 \pi-\tau_{j}\right)}
$$

The formula $\tan \left(\theta_{j}\right)$ can be used to solve for $\theta_{j}$. Using the following homogenous system from Chapter 4 ,

$$
\left[\begin{array}{ll}
G_{1} & G_{2} \\
H_{1} & H_{2}
\end{array}\right]\left[\begin{array}{c}
\cos \left(\tau_{j}\right) \\
\sin \left(\tau_{j}\right)
\end{array}\right]=\left[\begin{array}{l}
0 \\
0
\end{array}\right]
$$


$\cos \left(\tau_{j}\right)$ and $\sin \left(\tau_{j}\right)$ can be found with either of the two equations:

$$
\left(\cos \left(\tau_{j}\right), \sin \left(\tau_{j}\right)\right)=\left(\frac{-G_{2}}{\sqrt{G_{1}^{2}+G_{2}^{2}}}, \frac{G_{1}}{\sqrt{G_{1}^{2}+G_{2}^{2}}}\right)
$$

or

$$
\left(\cos \left(\tau_{j}\right), \sin \left(\tau_{j}\right)\right)=\left(\frac{-H_{2}}{\sqrt{H_{1}^{2}+H_{2}^{2}}}, \frac{H_{1}}{\sqrt{H_{1}^{2}+H_{2}^{2}}}\right)
$$

We have concluded by experimentation that it is significantly more numerically stable to use $H_{1}$ and $H_{2}$ because for half of the eigenvalues $G_{1}, G_{2} \approx 0$ at the frequencies we are trying to compute. To find the values of $\left\langle V_{j}, \cos (k x)\right\rangle$ and $\left\langle V_{j}, \sin (k x)\right\rangle, \int_{0}^{2 \pi} V_{j}(x) \cos (k x) d x$ and $\int_{0}^{2 \pi} V_{j} \sin (k x) d x$ must be computed analytically. Evaluating the integral $\int_{0}^{2 \pi} V_{j}(x) \cos (k x) d x$ first, we have the following:

$$
\begin{aligned}
\int_{0}^{2 \pi} V_{j}(x) \cos (k x) d x= & \int_{0}^{2 \pi \rho} A_{j} \cos \left(\omega_{j 1} x-\theta_{j}\right) \cos (k x) d x \\
& +\int_{2 \pi \rho}^{2 \pi} \cos \left(\omega_{j 2} x-\tau_{j}\right) \cos (k x) d x \\
= & \left.A_{j}\left[\frac{\sin \left(\left(k-\omega_{j 1}\right) x+\theta_{j}\right)}{2\left(k-\omega_{j 1}\right)}+\frac{\sin \left(\left(\omega_{j 1}+k\right) x-\theta_{j}\right)}{2\left(\omega_{j 1}+k\right)}\right]\right|_{0} ^{2 \pi \rho} \\
& +\left.\left[\frac{\sin \left(\left(k-\omega_{j 2}\right) x+\tau_{j}\right)}{2\left(k-\omega_{j 2}\right)}+\frac{\sin \left(\left(\omega_{j 2}+k\right) x-\tau_{j}\right)}{2\left(\omega_{j 2}+k\right)}\right]\right|_{2 \pi \rho} ^{2 \pi} \\
\left\langle V_{j}(x), \cos (k x)\right\rangle= & A_{j}\left[\frac{\sin \left(\left(k-\omega_{j 1}\right) 2 \pi \rho+\theta_{j}\right)}{2\left(k-\omega_{j 1}\right)}+\frac{\sin \left(\left(\omega_{j 1}+k\right) 2 \pi \rho-\theta_{j}\right)}{2\left(\omega_{j 1}+k\right)}\right. \\
& \left.-\frac{\sin \left(\theta_{j}\right)}{2\left(k-\omega_{j 1}\right)}-\frac{\sin \left(-\theta_{j}\right)}{2\left(\omega_{j 1}+k\right)}\right]+\frac{\sin \left(\left(k-\omega_{j 2}\right) 2 \pi+\tau_{j}\right)}{2\left(k-\omega_{j 2}\right)} \\
& +\frac{\sin \left(\left(\omega_{j 2}+k\right) 2 \pi-\tau_{j}\right)}{2\left(\omega_{j 2}+k\right)}+\frac{\sin \left(\left(k-\omega_{j 2}\right) 2 \pi \rho+\tau_{j}\right)}{2\left(k-\omega_{j 2}\right)} \\
& +\frac{\sin \left(\left(\omega_{j 2}+k\right) 2 \pi \rho-\tau_{j}\right)}{2\left(\omega_{j 2}+k\right)}
\end{aligned}
$$


Then evaluating the integral $\int_{0}^{2 \pi} V_{j} \sin (k x) d x$, we get an expression for $\left\langle V_{j}, \sin (k x)\right\rangle$ :

$$
\begin{aligned}
\int_{0}^{2 \pi} V_{j}(x) \sin (k x) d x= & \int_{0}^{2 \pi \rho} A_{j} \cos \left(\omega_{j 1} x-\theta_{j}\right) \sin (k x) d x \\
& +\int_{2 \pi \rho}^{2 \pi} \cos \left(\omega_{j 2} x-\tau_{j}\right) \sin (k x) d x \\
= & \left.A_{j}\left[-\frac{\cos \left(\left(k-\omega_{j 1}\right) x-\theta_{j}\right)}{2\left(k-\omega_{j 1}\right)}-\frac{\cos \left(\left(\omega_{j 1}+k\right) x-\theta_{j}\right)}{2\left(\omega_{j 1}+k\right)}\right]\right|_{0} ^{2 \pi \rho} \\
& +\left.\left[-\frac{\cos \left(\left(k-\omega_{j 2}\right) x+\tau_{j}\right)}{2\left(k-\omega_{j 2}\right)}-\frac{\cos \left(\left(\omega_{j 2}+k\right) x-\tau_{j}\right)}{2\left(\omega_{j 2}+k\right)}\right]\right|_{2 \pi \rho} ^{2 \pi} \\
\left\langle V_{j}, \sin (k x)\right\rangle= & A_{j}\left[-\frac{\cos \left(\left(k-\omega_{j 1}\right) 2 \pi \rho+\theta_{j}\right)}{2\left(k-\omega_{j 1}\right)}-\frac{\cos \left(\left(\omega_{j 1}+k\right) 2 \pi \rho-\theta_{j}\right)}{2\left(\omega_{j 1}+k\right)}\right. \\
& \left.+\frac{\cos \left(\theta_{j}\right)}{2\left(k-\omega_{j 1}\right)}+\frac{\cos \left(-\theta_{j}\right)}{2\left(\omega_{j 1}+k\right)}\right]-\frac{\cos \left(\left(k-\omega_{j 2}\right) 2 \pi+\tau_{j}\right)}{2\left(k-\omega_{j 2}\right)} \\
& -\frac{\cos \left(\left(\omega_{j 2}+k\right) 2 \pi-\tau_{j}\right)}{2\left(\omega_{j 2}+k\right)}-\frac{\cos \left(\left(k-\omega_{j 2}\right) 2 \pi \rho+\tau_{j}\right)}{2\left(k-\omega_{j 2}\right)} \\
& -\frac{\cos \left(\left(\omega_{j 2}+k\right) 2 \pi \rho-\tau_{j}\right)}{2\left(\omega_{j 2}+k\right)}
\end{aligned}
$$

Once $\left\langle V_{j}, \sin (k x)\right\rangle,\left\langle V_{j}, \cos (k x)\right\rangle$, and $\int_{0}^{2 \pi} V_{j}^{2} d x$ are evaluated and formulas for the parameters are computed, the heat equation with a discontinuous coefficient can be solved numerically. 


\section{Chapter 6}

Numerical Results

\subsection{Finding Eigenfunctions}

The following charts are the numerical results from approximating the $\omega_{j 2}$ frequency values numerically with secant method using the methods described in Chapter 4 . The charts report the theoretical frequency values, the approximated frequency values, the error between the two frequencies, and the number of iterations involved.

In the first trial, $\alpha_{1}=1, \alpha_{2}=2$, and $\rho=\frac{3}{8}$. The results are shown in Table 6.1.

\begin{tabular}{|r|r|r|r|r|}
\hline$j$ & $\lambda_{j}(\mathrm{eig})$ & $\lambda_{j}(S A K)$ & error & iterations \\
\hline 0 & 0.00000 & 0.00000 & $0.0000 \mathrm{e}+00$ & N/A \\
1 & 0.70612 & 0.70612 & $1.9065 \mathrm{e}-06$ & 7 \\
2 & 0.74970 & 0.74970 & $1.1742 \mathrm{e}-06$ & 10 \\
3 & 1.41365 & 1.41366 & $1.5047 \mathrm{e}-05$ & 6 \\
4 & 1.49759 & 1.49760 & $9.7335 \mathrm{e}-06$ & 8 \\
5 & 2.12401 & 2.12406 & $4.9602 \mathrm{e}-05$ & 5 \\
6 & 2.24195 & 2.24199 & $3.4769 \mathrm{e}-05$ & 9 \\
7 & 2.83862 & 2.83873 & $1.1357 \mathrm{e}-04$ & 6 \\
8 & 2.98124 & 2.98133 & $8.8403 \mathrm{e}-05$ & 9 \\
9 & 3.55870 & 3.55892 & $2.1187 \mathrm{e}-04$ & 5 \\
10 & 3.71434 & 3.71452 & $1.8605 \mathrm{e}-04$ & 8 \\
\hline
\end{tabular}

Table 6.1: Eigenvalues computed using eig, and using the method from Chapter 4, with $\alpha_{1}=1, \alpha_{2}=2$, and $\rho=3 / 8$

In the second trial, $\alpha_{1}=1, \alpha_{2}=2$, and $\rho=\frac{\sqrt{2}}{2 \pi}$. The results are shown in Table 6.2. In the third trial, $\alpha_{1}=1, \alpha_{2}=4, \rho=\frac{3}{8}$. The results are shown in Table 6.3.

In the fourth trial, $\alpha_{1}=4, \alpha_{2}=1, \rho=\frac{3}{8}$. The results are shown in Table 6.4.

\subsection{Results from Solving the PDE}

The initial data of the PDE in Figure 6.1 is the periodic function $\sin (2 x)$. As the time steps are increased, the solution behaves as expected and becomes more smooth as time increases. The figure 6.2 below illustrates to a PDE with discontinuous function as the initial data. As time increases, the function also behaves as expected and becomes more smooth over time. 


\begin{tabular}{|r|r|r|r|r|}
\hline$j$ & $\lambda_{j}(\mathrm{eig})$ & $\lambda_{j}(S A K)$ & error & iterations \\
\hline 0 & 0.00000 & 0.00000 & $1.9073 \mathrm{e}-06$ & N/A \\
1 & 0.75545 & 0.75538 & $6.9844 \mathrm{e}-05$ & 7 \\
2 & 0.88567 & 0.88508 & $5.8869 \mathrm{e}-04$ & 7 \\
3 & 1.54483 & 1.54425 & $5.8179 \mathrm{e}-04$ & 6 \\
4 & 1.71982 & 1.71931 & $5.0918 \mathrm{e}-04$ & 8 \\
5 & 2.39425 & 2.39255 & $1.7023 \mathrm{e}-03$ & 7 \\
6 & 2.49764 & 2.49754 & $9.9473 \mathrm{e}-05$ & 7 \\
7 & 3.24999 & 3.28299 & $3.3005 \mathrm{e}-02$ & 7 \\
8 & 3.28558 & 3.28299 & $2.5892 \mathrm{e}-03$ & 8 \\
9 & 4.01042 & 4.01004 & $3.8098 \mathrm{e}-04$ & 7 \\
10 & 4.16204 & 4.15984 & $2.1999 \mathrm{e}-03$ & 8 \\
\hline
\end{tabular}

Table 6.2: Eigenvalues computed using eig, and using the method from Chapter 4, with $\alpha_{1}=1, \alpha_{2}=2$, and $\rho=\sqrt{2} /(2 \pi)$

\begin{tabular}{|r|r|r|r|r|}
\hline$j$ & $\lambda_{j}$ (eig) & $\lambda_{j}(S A K)$ & error & iterations \\
\hline 0 & 0.00000 & 0.00000 & $0.0000 \mathrm{e}+00$ & N/A \\
1 & 0.38777 & 0.38777 & $1.6893 \mathrm{e}-06$ & 10 \\
2 & 0.56697 & 0.56697 & $1.9322 \mathrm{e}-06$ & 6 \\
3 & 0.88006 & 0.88007 & $1.0664 \mathrm{e}-05$ & 10 \\
4 & 0.98041 & 0.98043 & $2.0143 \mathrm{e}-05$ & 7 \\
5 & 1.36014 & 1.36019 & $5.9469 \mathrm{e}-05$ & 7 \\
6 & 1.48804 & 1.48808 & $3.6118 \mathrm{e}-05$ & 7 \\
7 & 1.78815 & 1.81818 & $3.0028 \mathrm{e}-02$ & 8 \\
8 & 1.95589 & 1.95604 & $1.5238 \mathrm{e}-04$ & 7 \\
9 & 2.33935 & 2.33965 & $2.9489 \mathrm{e}-04$ & 6 \\
10 & 2.37468 & 2.37481 & $1.3570 \mathrm{e}-04$ & 9 \\
\hline
\end{tabular}

Table 6.3: Eigenvalues computed using eig, and using the method from Chapter 4, with $\alpha_{1}=1, \alpha_{2}=4$, and $\rho=3 / 8$ 


\begin{tabular}{|r|r|r|r|r|}
\hline$j$ & $\lambda_{j}(\mathrm{eig})$ & $\lambda_{j}(S A K)$ & error & iterations \\
\hline 0 & 0.00000 & 0.00000 & $0.0000 \mathrm{e}+00$ & N/A \\
1 & 1.12098 & 1.12098 & $2.9090 \mathrm{e}-06$ & 12 \\
2 & 1.53831 & 1.53832 & $4.8667 \mathrm{e}-06$ & 7 \\
3 & 2.53441 & 2.54545 & $1.1045 \mathrm{e}-02$ & 14 \\
4 & 3.04544 & 3.04547 & $3.5498 \mathrm{e}-05$ & 6 \\
5 & 4.05027 & 4.05037 & $1.0368 \mathrm{e}-04$ & 10 \\
6 & 4.42367 & 4.42376 & $9.0057 \mathrm{e}-05$ & 7 \\
7 & 5.50057 & 5.59034 & $8.9778 \mathrm{e}-02$ & 9 \\
8 & 5.59008 & 5.59034 & $2.6345 \mathrm{e}-04$ & 7 \\
9 & 6.67457 & 6.90909 & $2.3452 \mathrm{e}-01$ & 10 \\
10 & 7.12572 & 7.12625 & $5.2723 \mathrm{e}-04$ & 7 \\
\hline
\end{tabular}

Table 6.4: Eigenvalues computed using eig, and using the method from Chapter 4, with $\alpha_{1}=4, \alpha_{2}=1$, and $\rho=3 / 8$
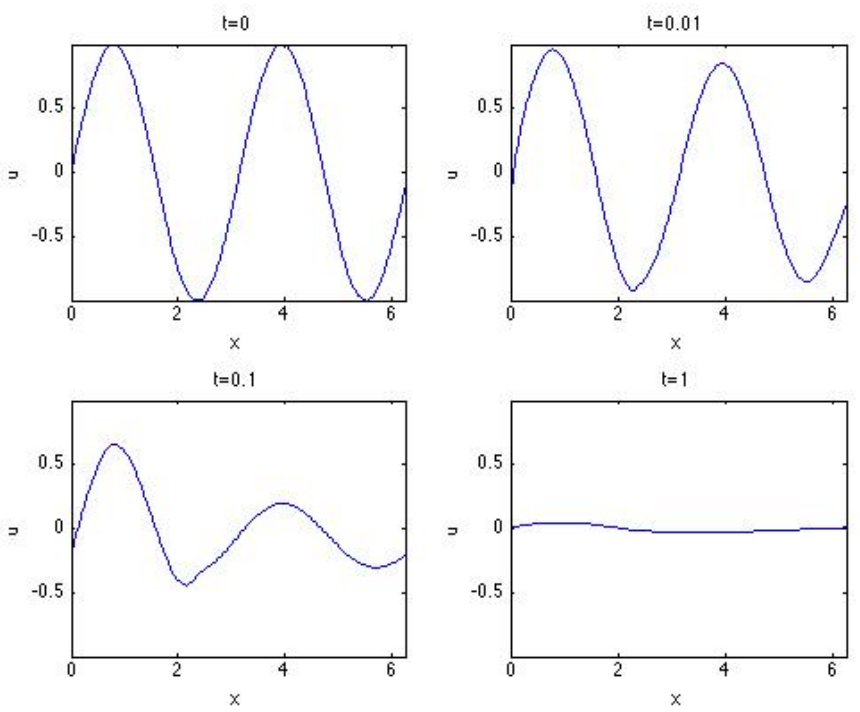

Figure 6.1: Solution with $\alpha_{1}=1, \alpha_{2}=2, \rho=3 / 8$ and $u(x, 0)=\sin (2 x)$ 

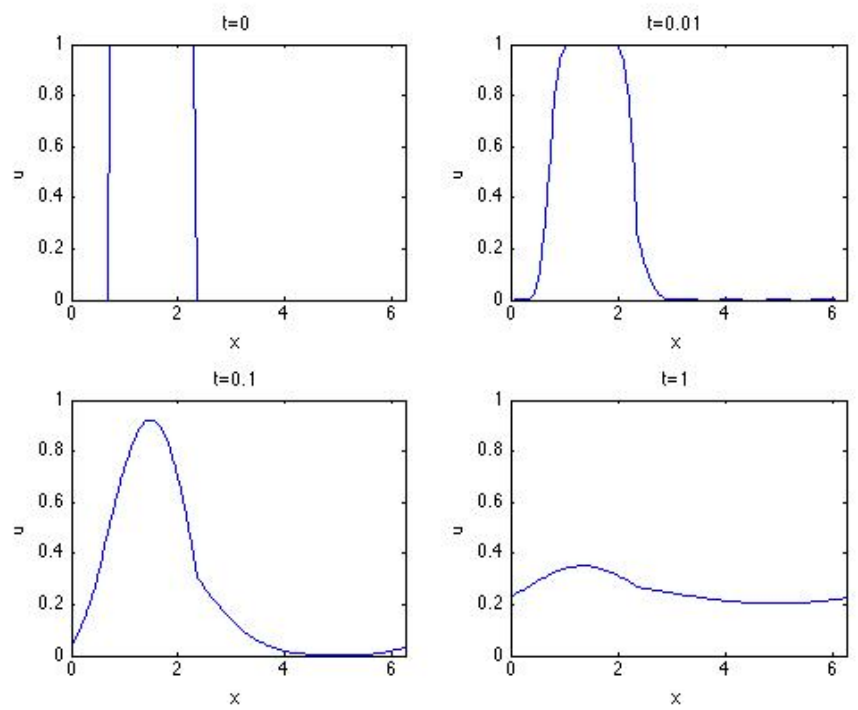

Figure 6.2: Solution with $\alpha_{1}=1, \alpha_{2}=2, \rho=3 / 8$ and $u(x, 0)$ is a characteristic function 


\section{Chapter 7}

\section{Conclusion}

The outcome of this project was overall successful. An accurate and efficient algorithm for computing eigenfunctions of differential operators was used to model the heat diffusion through two homogenous materials was designed and implemented. This method substantially simplifies the solution of such models with discontinuous coefficients and bypasses the limitations of analytical methods and numerical methods; however, there is future work that can be done with this project to improve and expand it.

By solving problems with discontinuous coefficients accurately and efficiently, the method can substantially aid researchers in science and engineering who need to simulate such heat diffusion processes. To improve this method, a more reliable way to find the zeros of the determinant should be explored. Alternatively, working with the smallest singular value of the matrix should be investigated because it is a more reliable indicator of distance to the nearest non-invertible matrix than the determinant is. For expansion, this project can be investigated past one dimensional PDE with discontinuous coefficients; furthermore, the case of more than two homogeneous materials could be investigated. This could improve insight on different types of equations with discontinuous coefficients and allow for more research on their qualitative behaviors. This project could also could be continued by studying the behavior of these equations in two dimensional or three dimensional models. 


\section{Appendix A}

Code

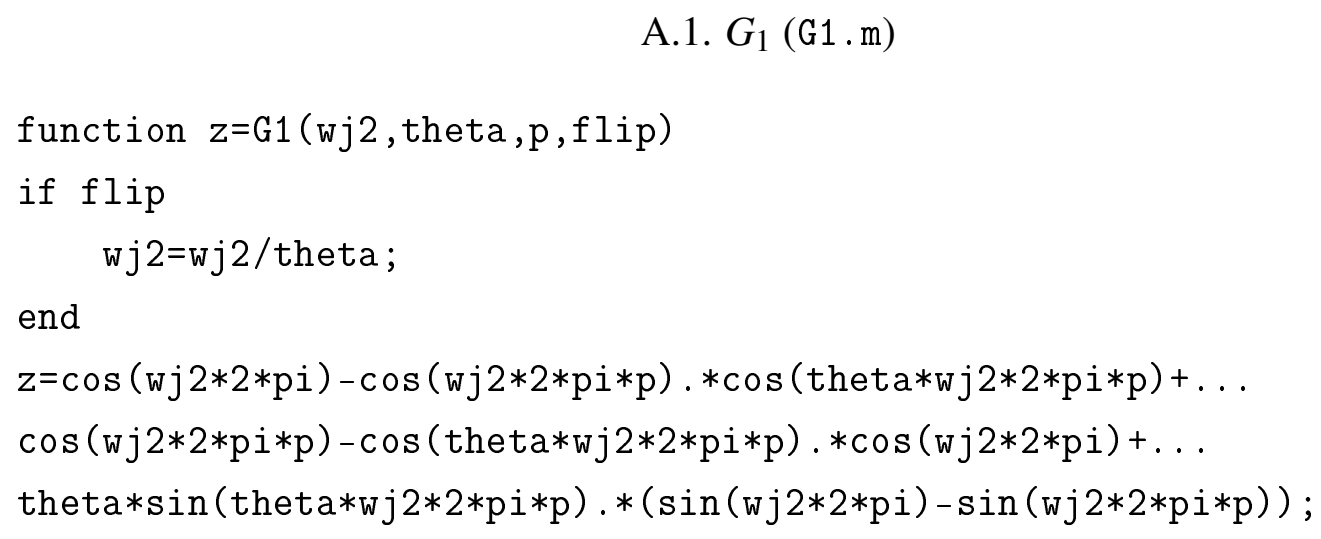

G1. $\mathrm{m}$ is used to generate the coefficient of $\cos \left(\tau_{j}\right), G_{1}$.

$$
\text { A.2. } G_{2}(\mathrm{G} 2 . \mathrm{m})
$$

function $z=G 2(w j 2$, theta, $p, f l i p)$

if flip

$$
\text { w } j 2=w j 2 / \text { theta }
$$

end

$z=\sin (w j 2 * 2 * p i)-\sin (w j 2 * 2 * p i * p) . * \cos ($ theta $* w j 2 * 2 * p i * p)+\ldots$

$\sin ($ w $2 * 2 *$ pi $*$ p) $-\cos ($ theta*wj $2 * 2 *$ pi $*$ p) . *sin $(w j 2 * 2 *$ pi $)-\ldots$

theta*sin (theta*wj $2 * 2 * p i * p) . *(\cos (w j 2 * 2 * p i)-\cos (w j 2 * 2 * p i * p))$;

G2. $\mathrm{m}$ is used to generate the coefficient of $\sin \left(\tau_{j}\right), G_{2}$.

$$
\text { A.3. } H_{1}(\mathrm{H} 1 . \mathrm{m})
$$

function $z=H 1(w j 2$, theta,p,flip)

if flip

$$
\text { wj } 2=w j 2 / \text { theta; }
$$

end

$z=-\cos (w j 2 * 2 * p i)+\cos ($ theta $* w j 2 * 2 * p i * p) . * \cos (w j 2 * 2 * p i * p)+\ldots$

theta*sin $(w j 2 * 2 *$ pi $*$ p) . *sin (theta*wj $2 * 2 *$ pi*p); 
H1. $\mathrm{m}$ is used to generate the coefficient of $\cos \left(\tau_{j}\right), H_{1}$.

\section{A.4. $H_{2}(\mathrm{H} 2 . \mathrm{m})$}

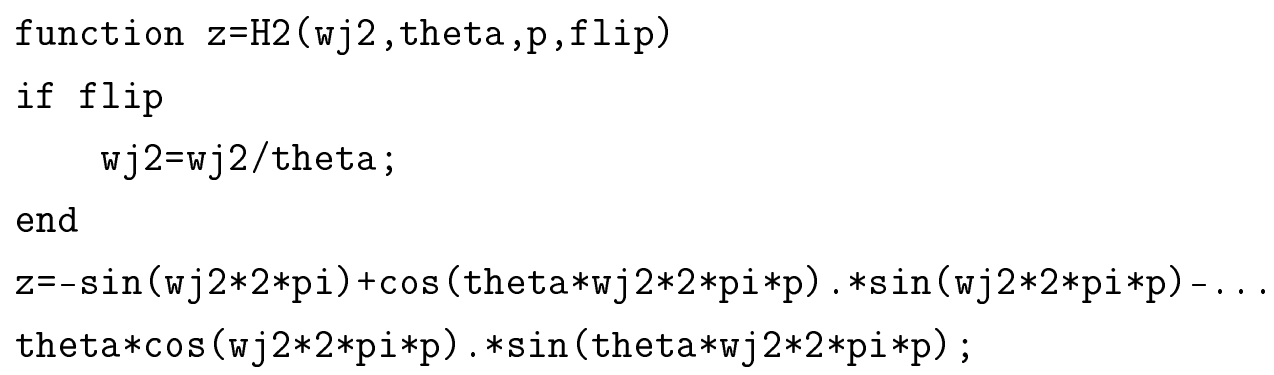

$\mathrm{H} 2 . \mathrm{m}$ is used to generate the coefficient of $\sin \left(\tau_{j}\right), H_{2}$.

\section{A.5. Fourier Transform (myfft.m)}

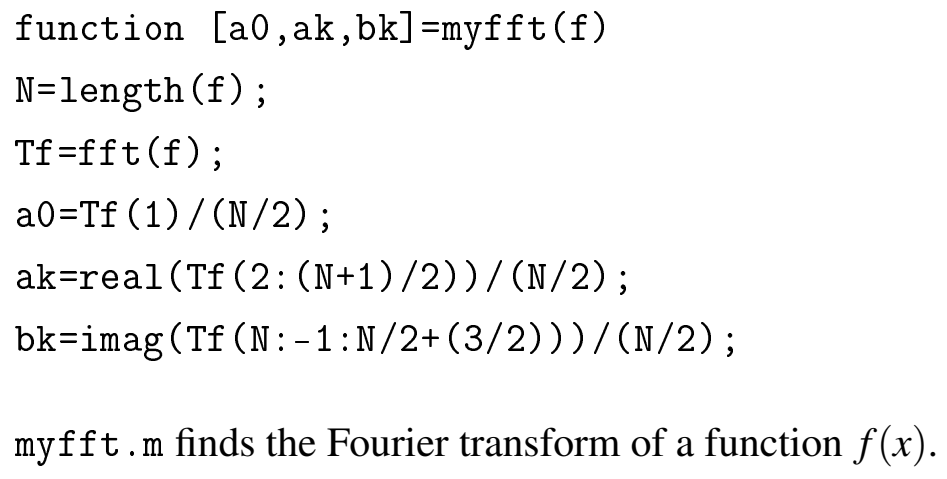

A.6. Solving for $U_{j}$ (finalform.m)

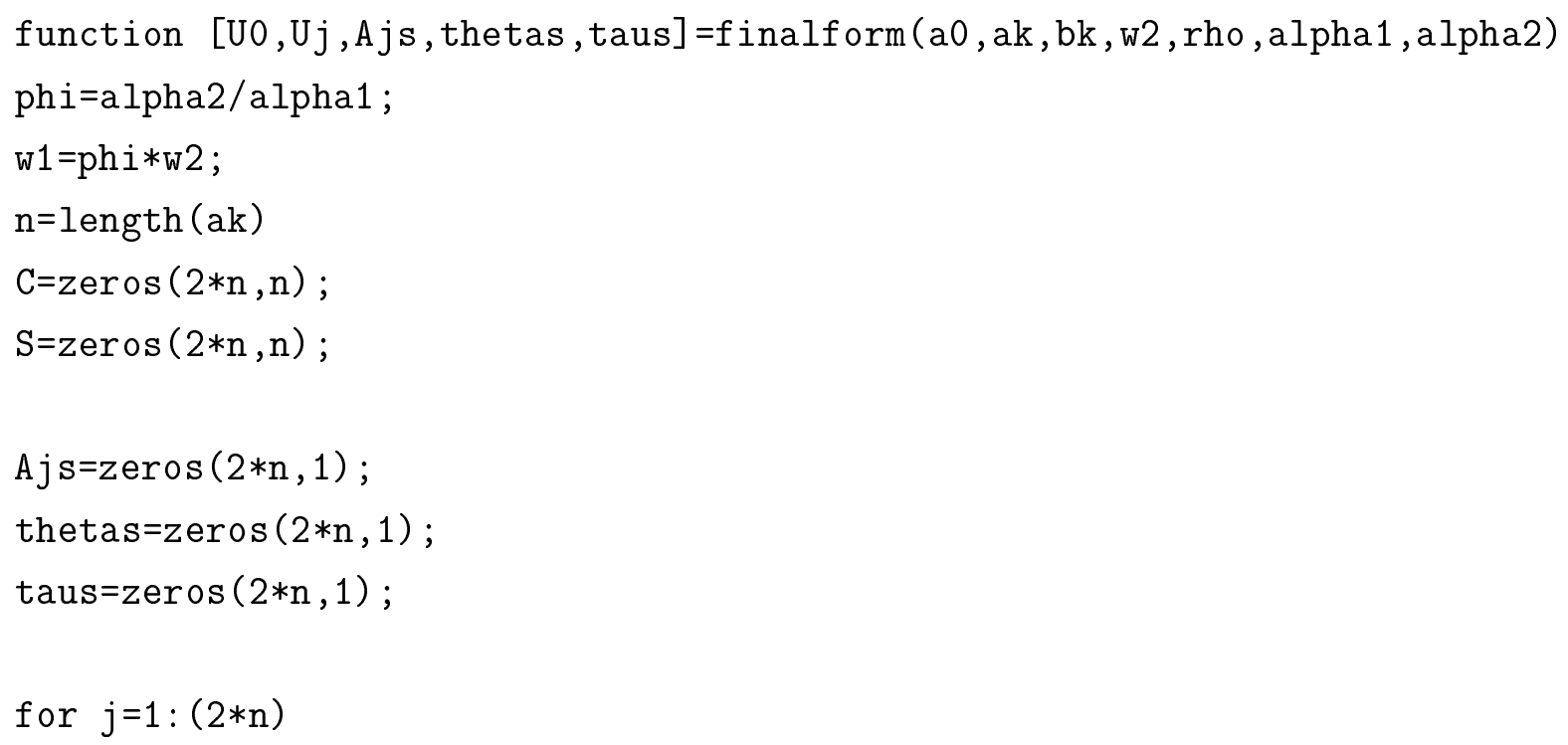


$w j 2=w 2(j)$;

$w j 1=w 1(j)$;

g2 j=H2 (wj2, phi , rho , 0);

g1j=H1 (wj2, phi , rho , 0);

$r j=\operatorname{sqrt}((g 1 j) \sim 2+(g 2 j) \sim 2)$;

ctauj=-g2j/rj;

stauj=g1j/rj;

$\operatorname{tau}=\operatorname{atan} 2(\operatorname{stauj}, \mathrm{ctauj})$;

$A j=\operatorname{sqrt}((\cos (w j 2 * 2 *$ pi-tau $))-2+(((\cos (w j 2 * 2 *$ pi $*$ rho-tau $)-$

$(\cos ($ wj $1 * 2 *$ pi $*$ rho $) * \cos (w j 2 * 2 *$ pi-tau $))) / \sin (w j 1 * 2 *$ pi*rho $)))-2)$;

theta $=\operatorname{atan} 2((\cos (w j 2 * 2 *$ pi $*$ rho - tau $)-\cos ($ wj $1 * 2 *$ pi $*$ rho $) *$ $\cos ($ wj $2 * 2 *$ pi-tau $)) /(\sin ($ wj $1 * 2 *$ pi $*$ rho $)), \cos (w j 2 * 2 *$ pi-tau $))$;

$\operatorname{Ajs}(j)=A j$;

$\operatorname{thetas}(j)=$ theta;

$\operatorname{taus}(j)=\operatorname{tau}$;

for $k=1: n$

$$
\begin{aligned}
& \mathrm{C}(\mathrm{j}, \mathrm{k})=\mathrm{Aj} *(((\sin ((\mathrm{k}-\mathrm{wj} 1) * 2 * \mathrm{pi} * \operatorname{rho}+\text { theta }) /(2 *(\mathrm{k}-\mathrm{wj} 1)))+ \\
& (\sin ((w j 1+\mathrm{k}) * 2 * \text { pi } * \text { rho-theta }) /(2 *(w j 1+\mathrm{k})))- \\
& (\sin (\text { theta }) /(2 *(\mathrm{k}-\mathrm{wj} 1)))-(\sin (-\operatorname{theta}) /(2 *(w j 1+\mathrm{k}))))) \ldots \\
& +(\sin (2 * \mathrm{pi} *(\mathrm{k}-\mathrm{wj} 2)+\mathrm{tau})-\sin (2 * \mathrm{pi} * \mathrm{rho} *(\mathrm{k}-\mathrm{wj} 2)+\mathrm{tau})) / \ldots \\
& (2 *(\mathrm{k}-\mathrm{wj} 2))+(\sin (2 * \mathrm{pi} *(\mathrm{k}+\mathrm{wj} 2)-\mathrm{tau})-\ldots \\
& \sin (2 * \mathrm{pi} * \operatorname{rho} *(\mathrm{k}+\mathrm{wj} 2)-\mathrm{tau})) /(2 *(\mathrm{k}+\mathrm{wj} 2)) \text {; } \\
& \mathrm{S}(\mathrm{j}, \mathrm{k})=\mathrm{Aj} *(((-\cos ((\mathrm{k}-\mathrm{wj} 1) * 2 * \mathrm{pi} * \mathrm{rho}+\text { theta }) /(2 *(\mathrm{k}-\mathrm{wj} 1)))- \\
& (\cos ((w j 1+\mathrm{k}) * 2 * \text { pi } * \text { rho-theta }) /(2 *(w j 1+\mathrm{k})))+ \\
& (\cos (\text { theta }) /(2 *(k-w j 1)))+(\cos (- \text { theta }) /(2 *(w j 1+k))))) \ldots \\
& +(-\cos (2 * \mathrm{pi} *(\mathrm{k}-\mathrm{w} \mathrm{j} 2)+\mathrm{tau})+\cos (2 * \mathrm{p} i * r h o *(\mathrm{k}-\mathrm{wj} 2)+\mathrm{tau})) / \ldots \\
& (2 *(\mathrm{k}-\mathrm{wj} 2))+(-\cos (2 * \mathrm{p} i *(\mathrm{k}+\mathrm{wj} 2)-\mathrm{tau})+\ldots \\
& \cos (2 * \mathrm{pi} * \operatorname{rho} *(\mathrm{k}+\mathrm{wj} 2)-\mathrm{tau})) /(2 *(\mathrm{k}+\mathrm{wj} 2)) \text {; }
\end{aligned}
$$

end

$$
\begin{aligned}
& Z(j, 1)=((A j)-2 / 2) *(2 * \text { pi } * \text { rho }+(\sin (4 * w j 1 * \text { pi } * \text { rho }-2 * \text { theta }) /(2 * w j 1))- \\
& (\sin (-2 * \text { theta }) /(2 * w j 1)))+(p i+(\sin (4 * w j 2 * p i-2 * \operatorname{tau}) / \ldots \\
& (4 * w j 2)))-(\text { pi } * \text { rho }+(\sin (4 * w j 2 * \text { pi } * \text { rho }-2 * t a u) /(4 * w j 2))) \text {; }
\end{aligned}
$$


end

$\mathrm{U} 0=\mathrm{a} 0 / 2$;

$\mathrm{Uj}=(1 . / \mathrm{Z}) \cdot *(\mathrm{C} * \mathrm{ak}+\mathrm{S} * \mathrm{bk})$;

The function finalform.m uses the coefficients for the Fourier series that were produced by the Fourier transform in myfft.m to represent the Fourier series in the correct form.

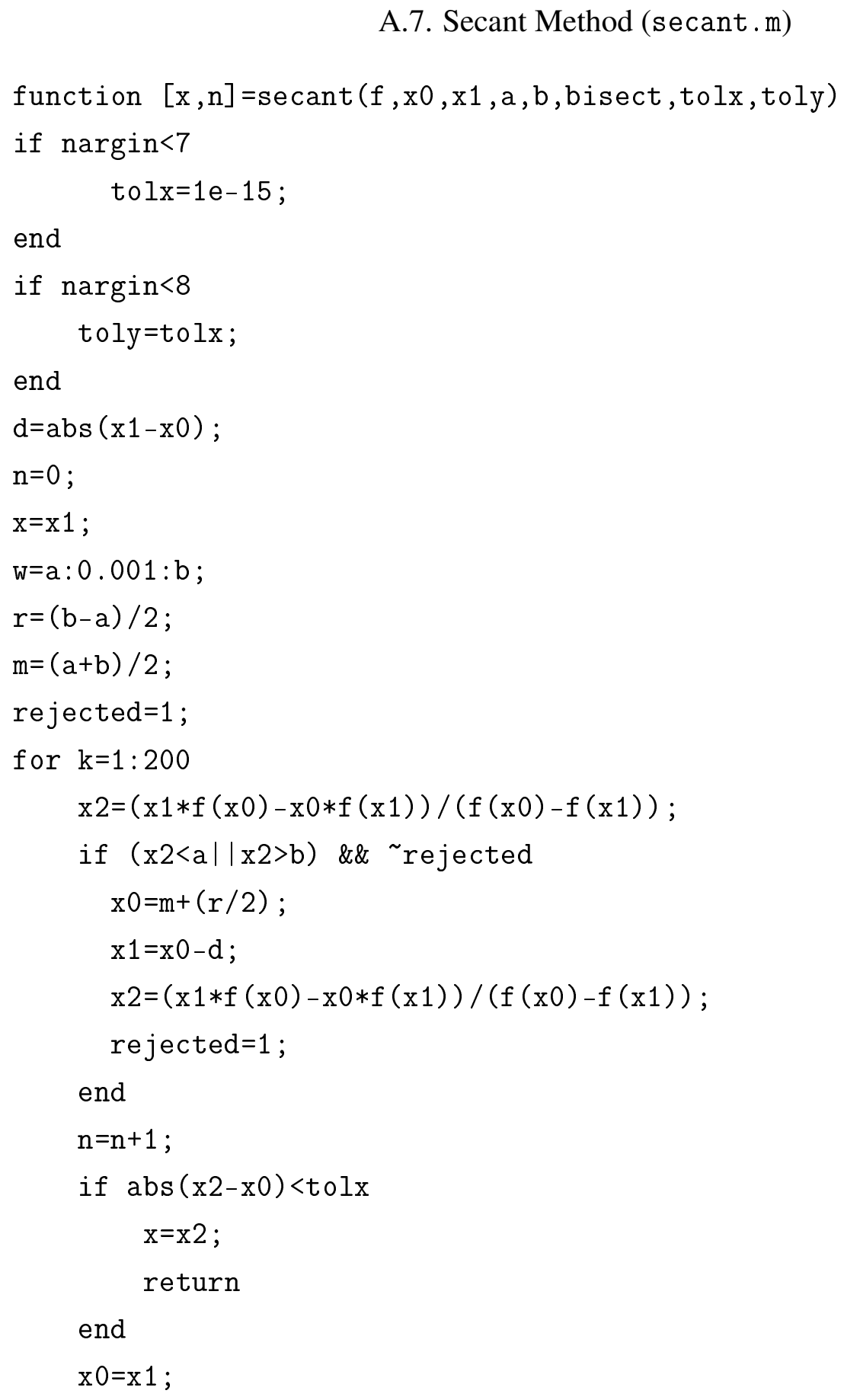


$\mathrm{x} 1=\mathrm{x} 2 ;$

end

The function secant.m produces an approximate root of a given function $f(x)$ using the secant method.

\section{A.8. Finding $\omega_{j 2}$ Frequencies (getw2.m)}

function [w2s]=getw2 (rho, alpha1, alpha2,n)

phi=alpha2/alpha1;

$\operatorname{sak}=1 /(1+r h o *(p h i-1))$;

flip=0;

if flip sak=sak*phi;

end

$\mathrm{w} 2 \mathrm{~s}=[\mathrm{]}$;

$c=0.05$;

$j=1$;

while length $($ w $2 \mathrm{~s})<\mathrm{n}$

$\mathrm{w} 0=\operatorname{sak} * j$;

[w1] = secant $(@(x)$ findeigs ( $x, p h i, r h o, f l i p), w 0, w 0+c * s a k, \ldots$ sak* $(j-(3 / 4)), \operatorname{sak} *(j+(3 / 4)), 1,1 e-8)$;

if $\operatorname{abs}(\sin (\operatorname{phi} * \mathrm{w} 1 * 2 * \mathrm{pi} * \mathrm{rho}))>1 \mathrm{e}-10$

$\mathrm{w} 2 \mathrm{~s}=[\mathrm{w} 2 \mathrm{~s} ; \mathrm{w} 1]$;

else

disp ([ 'rejected: ' num2str(w1) ])

end

if $\mathrm{w} 1<\mathrm{w} 0$

$\mathrm{a}=\mathrm{j} * \mathrm{sak}$;

$\mathrm{b}=(j+(3 / 4)) * \mathrm{sak}$;

else

$a=(j-(3 / 4)) * s a k$;

$b=j * s a k$;

end

w0 $=2 *$ w0 $0-$ w 1 ; 


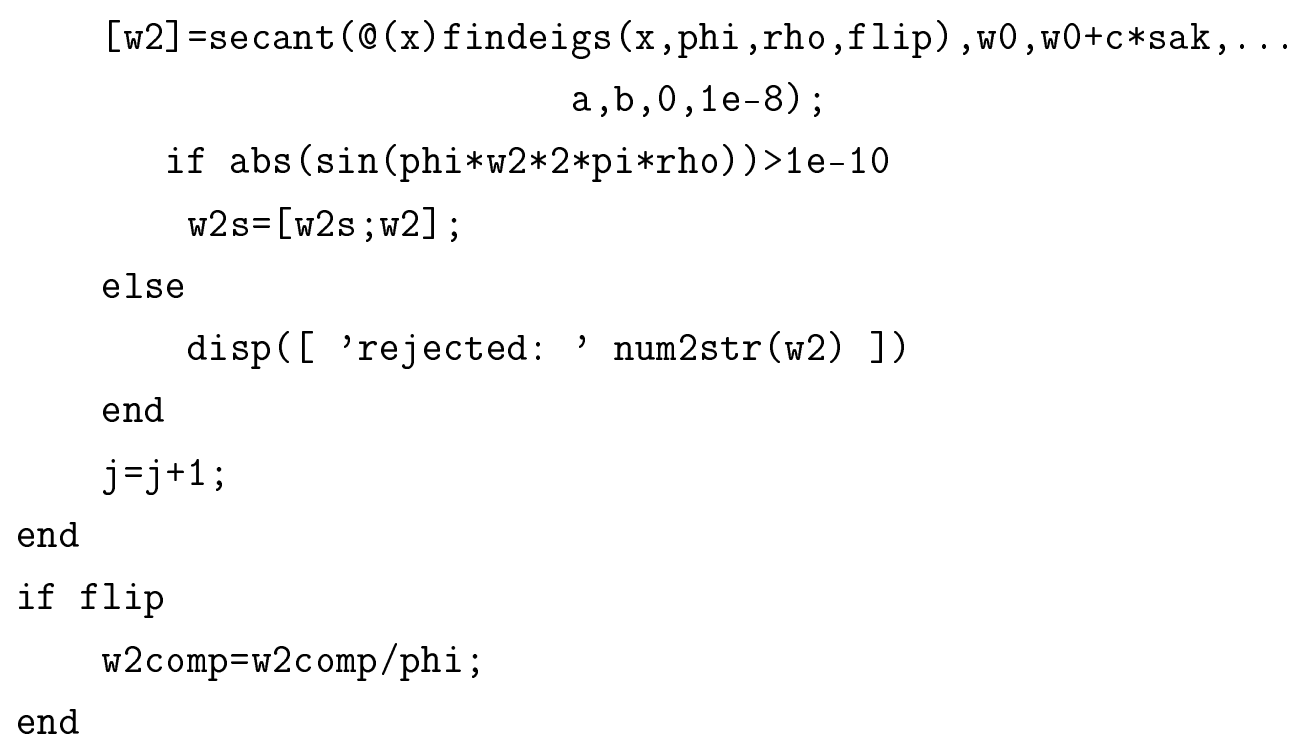

The function getw2.m employs the secant method to find the $\omega_{j 2}$ frequencies.

A.9. Finding Eigenvalues (findeigs.m)

function $z=f$ indeigs ( $w j 2$, theta,p,flip, w0)

$z=G 1(w j 2$, theta $, p, f l i p) . * H 2(w j 2$, theta $, p, f l i p)-\ldots$

H1 (wj2, theta, p, flip) .*G2 (wj2, theta, p, flip);

if nargin $>4$

$$
z=z /(w j 2-w 0) \text {; }
$$

end

The function findeigs .m outputs eigenvalues with the input of $\omega_{j 2}$ frequencies, $\theta_{j}$ phase shifts, and the point of discontinuity $\rho$.

A.10. Solve PDE (solvepde.m)

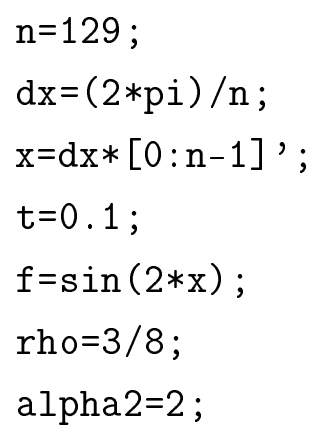




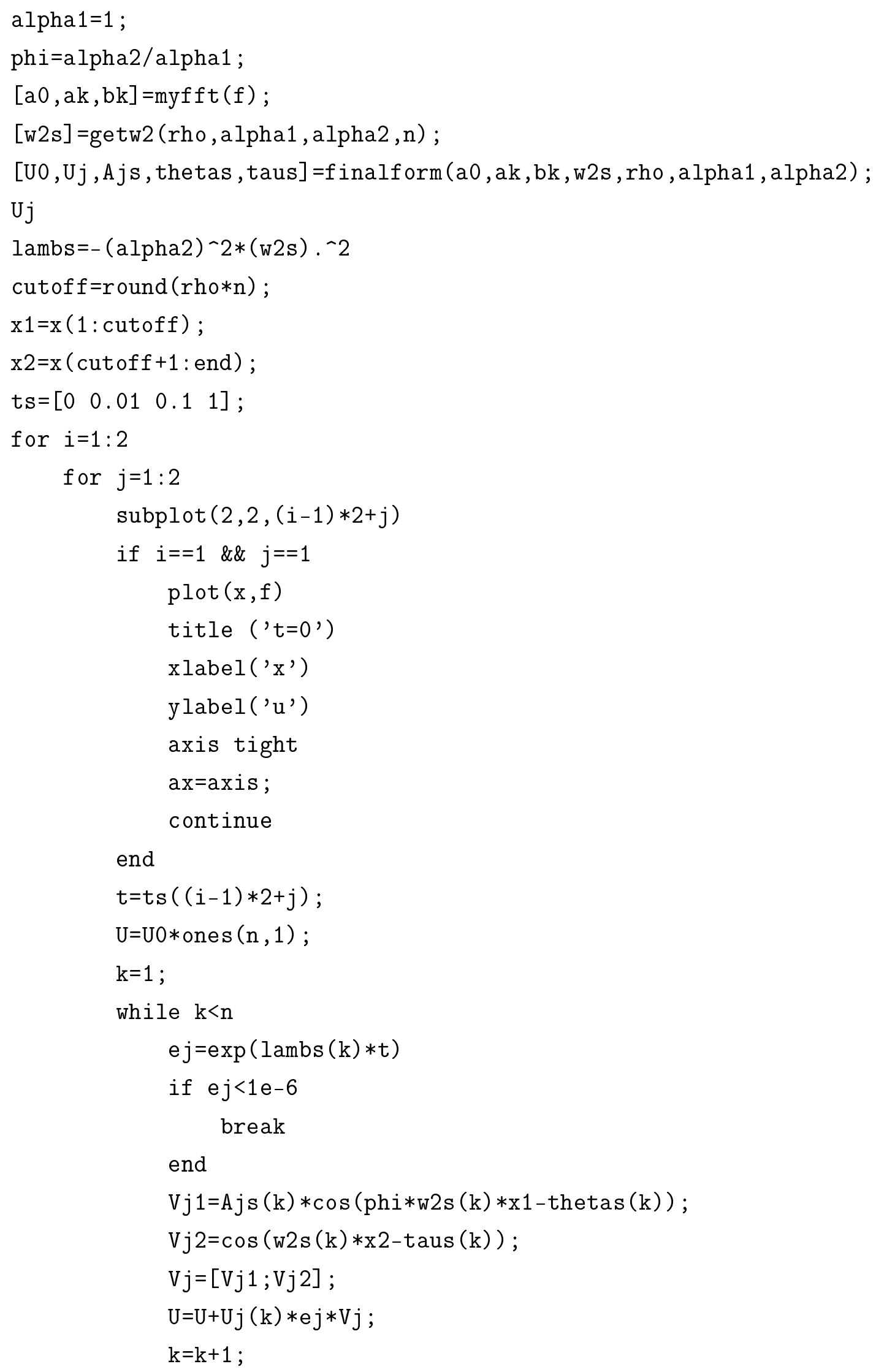




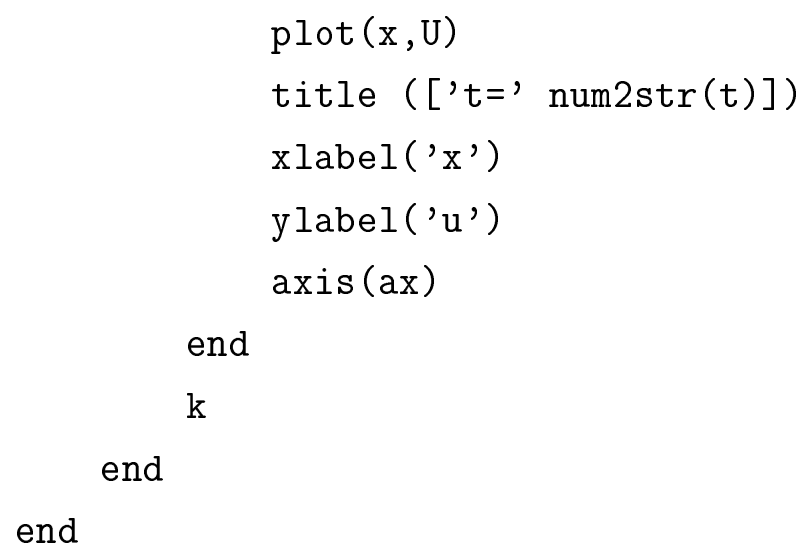

The function solvepde.m employs myfft.m, finalform.m, and getw2.m to find the necessary parameters and then finally outputs the solution to the PDE. 
Bibliography

[1] Arfken, G. B., Weber, H. J. and Harris, F. (2012) Mathematical Methods for Physicists: A Comprehensive Guide, 7th Edition. Waltham, MA: Academic Press.

[2] Burden, R. L. and Faires, J. D. (2010) Numerical Analysis, 9th Edition. Stamford, CT: Cengage Learning.

[3] Farlow, S. J. (1993) Partial Differential Equations for Scientists and Engineers. New York, NY: Dover Publications, Inc.

[4] Fefferman, C. (1983) The Uncertainty Principle. Bulletin of the American Mathematical Society 9, 129-206.

[5] Filoche, M., Mayaboroda S. and B. Patterson, Localization of eigenfunctions of a one-dimensional elliptic operator', Contemporary Mathematics 581 (2012), 99-116.

[6] Gelb, A. and Tanner, J. (2006) Robust reprojection methods for the resolution of the Gibbs phenomenon. Applied Computational Harmonic Analysis 20, 3-25.

[7] Golub, G. H., Meurant, G.: Matrices, Moments and Quadrature.Proceedings of the 15th Dundee Conference, June-July 1993, Griffiths, D. F., Watson, G. A. (eds.), Longman Scientific \& Technical (1994)

[8] Golub, G. H. and van Loan, C. (1996) Matrix Computations. Baltimore, MD: Johns Hopkins University Press.

[9] Gustafsson, B., Kreiss, H.-O. and Oliger, J. (1995) Time Dependent Problems And Difference Methods. New York, NY: John Wiley \& Sons, Inc.

[10] Lambers, J. V. (2012) Approximate Diagonalization of Variable-Coefficient Differential Operators Through Similarity Transformations. Computers and Mathematics with Applications 64(8), 2575-2593.

[11] Lambers, J. V. (2008) Enhancement of Krylov Subspace Spectral Methods by Block Lanczos Iteration. Electronic Transactions on Numerical Analysis 28, 89-106. 